\title{
VANISHING IDEALS OVER ODD CYCLES
}

\author{
M. Eduardo Uribe-Paczka, Eliseo Sarmiento and Carlos Rentería \\ Márquez
}

\begin{abstract}
Let $K$ be a finite field. Let $X^{*}$ be a subset of the affine space $K^{n}$, which is parameterized by odd cycles. In this paper we give an explicit Gröbner basis for the vanishing ideal, $\mathbf{I}\left(X^{*}\right)$, of $X^{*}$. We give an explicit formula for the regularity of $\mathbf{I}\left(X^{*}\right)$ and finally if $X^{*}$ is parameterized by an odd cycle of length $k$, we show that the Hilbert function of the vanishing ideal of $X^{*}$ can be written as linear combination of Hilbert functions of degenerate torus.
\end{abstract}

\section{Introduction}

We introduce some basic notions from coding theory. Let $K=\mathbb{F}_{q}$ be the finite field with $q$ elements. We consider the $n$-dimensional vector space $\mathbb{F}_{q}^{n}$ whose elements are $n$-tuples $a=\left(a_{1}, \ldots, a_{n}\right)$ with $a_{i} \in \mathbb{F}_{q}$.

A linear code $C$ over the alphabet $\mathbb{F}_{q}$ is a linear subspace of $\mathbb{F}_{q}^{n}$. The elements of $C$ are called codewords. We call $n$ the length of the code $C$ and $\operatorname{dim}_{\mathbb{F}_{q}} C$ the dimension of the code $C$ as an $\mathbb{F}_{q}$-vector space. The weight of an element $a=\left(a_{1}, \ldots, a_{n}\right) \in \mathbb{F}_{q}^{n}$ is defined as $w(a)=\left|\left\{i \mid a_{i} \neq 0\right\}\right|$. The minimum distance $\delta(C)$ of a code $C \neq 0$ is given by:

$$
\delta(C)=\min \{w(a) \mid 0 \neq a \in C\} .
$$

Key Words: Toric set, Parameterized code, Hilbert function, Vanishing Ideal, Regularity index.

2010 Mathematics Subject Classification: Primary 13P25; Secondary 14G50, 14G15, 11T71, 94B27, 94B05.

Received: 12.09 .2018

Accepted: 19.10 .2018 
Recall that the projective space of dimension $s-1$ over $K$, denoted by $\mathbb{P}_{K}^{s-1}$, is the quotient space

$$
\left(K^{s} \backslash\{0\}\right) / \sim
$$

where two points $\alpha, \beta$ in $K^{s} \backslash\{0\}$ are equivalent if $\alpha=\lambda \beta$ for some $\lambda \in K$. We denote the equivalence class of $\alpha$ by $[\alpha]$. Let $S=K\left[t_{1}, \ldots, t_{s}\right]=\oplus_{d=0}^{\infty} S_{d}$ be a polynomial ring over the field $K$ with the standard grading. Let $\mathbb{Y}$ be a subset of $\mathbb{P}_{K}^{s-1}$, where $\mathbb{P}_{K}^{s-1}$ is a projective space over the field $K$. Fix a degree $d \geq 1$. Let $P_{1}, \ldots, P_{m}$ be a set of representatives for the points of $\mathbb{Y}$ with $m=|\mathbb{Y}|$. For each $i$ there is $f_{i} \in S_{d}$ such that $f_{i}\left(P_{i}\right) \neq 0$. Let $P_{i}=\left[\left(a_{1}, \ldots, a_{s}\right)\right]$, there is at least one $j$ in $\{1, \ldots, s\}$ such that $a_{j} \neq 0$. Setting $f_{i}\left(t_{1}, \ldots, t_{s}\right)=t_{j}^{d}$ one has that $f_{i} \in S_{d}$ and $f_{i}\left(P_{i}\right) \neq 0$. The evaluation map, denoted by ev , is defined as:

$$
\mathrm{ev}_{d}: S_{d}=K\left[t_{1}, \ldots, t_{s}\right]_{d} \rightarrow K^{|\mathbb{Y}|}, \quad f \mapsto\left(\frac{f\left(P_{1}\right)}{f_{1}\left(P_{1}\right)}, \ldots, \frac{f\left(P_{m}\right)}{f_{m}\left(P_{m}\right)}\right)
$$

The map $\mathrm{ev}_{d}$ is well-defined, i.e., it is independent of the set of represen-

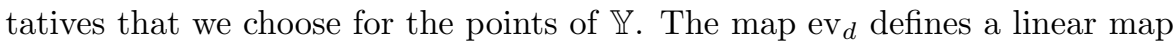
of $K$-vector spaces. The image of $S_{d}$ under ev $d$, denoted by $C_{\mathbb{Y}}(d)$, is called a projective Reed-Muller-type code of degree $d$ over $\mathbb{Y}[5,10]$. It is also called an evaluation code associated to $\mathbb{Y}$.

Let $Y$ be a subset of $K^{s}$, and let $\mathbb{Y}$ be the projective closure of $Y$. As $Y$ is finite, its projective closure is:

$$
\mathbb{Y}=\{[(1, \alpha)] \mid \alpha \in Y\} \subset \mathbb{P}_{K}^{s} .
$$

Let $P_{1}, \ldots, P_{m}$ be the points of $Y$, and let $S_{\leq d}$ be the $K$-vector space of all polynomials of $S$ of degree at most $d$. The evaluation map

$$
\mathrm{ev}_{d}^{a}: S_{\leq d} \longrightarrow K^{|Y|}, \quad f \mapsto\left(f\left(P_{1}\right), \ldots, f\left(P_{m}\right)\right),
$$

defines a linear map of $K$-vector spaces. The image of $\operatorname{ev}_{d}^{a}$, denoted by $C_{Y}(d)$, defines a linear code. We call $C_{Y}(d)$ the affine Reed-Muller-type code of degree $d$ on $Y$.

Let $y^{v_{1}}, \ldots, y^{v_{s}}$ be a finite set of monomials. As usual if $v_{i}=\left(v_{i 1}, \ldots, v_{i n}\right) \in$ $\mathbb{N}^{n}$, then we set

$$
y^{v_{i}}=y_{1}^{v_{i 1}} \cdots y_{n}^{v_{i n}}, \quad i=1, \ldots, s,
$$

where $y_{1}, \ldots, y_{n}$ are the indeterminates of a ring of polynomials with coefficients in $K$. Consider the following set parameterized by these monomials

$$
X^{*}:=\left\{\left(x_{1}^{v_{11}} \cdots x_{n}^{v_{1 n}}, \ldots, x_{1}^{v_{s 1}} \cdots x_{n}^{v_{s n}}\right) \in K^{s} \mid x_{i} \in K^{*} \text { for all } i\right\}
$$


where $K^{*}=K \backslash\{0\}$. Following [15] we call $X^{*}$ an affine algebraic toric set parameterized by $y^{v_{1}}, \ldots, y^{v_{s}}$. The set $X^{*}$ is a multiplicative group under componentwise multiplication. Following [15] we call $C_{X^{*}}(d)$ a parameterized affine code of degree $d$. Parameterized affine codes are special types of affine Reed-Muller codes in the sense of [21, p. 37]. If $s=n=1$ and $v_{1}=1$, then $X^{*}=\mathbb{F}_{q}^{*}$ and we obtain the classical Reed-Solomon code of degree $d$ [20, p. 42]. Some families of evaluation codes have been studied extensively, including several variations of Reed-Muller codes $[4,5,9,11,10,18]$.

The dimension and length of $C_{X^{*}}(d)$ are given by $\operatorname{dim}_{K} C_{X^{*}}(d)$ and $\left|X^{*}\right|$ respectively. The dimension and length are two of the basic parameters of a linear code, the third basic parameter is the minimum distance. The minimum distance of $C_{X^{*}}(d)$ will be denoted by $\delta_{d}$. The basic parameters of $C_{X^{*}}(d)$ are related by the Singleton bound for the minimum distance

$$
\delta_{d} \leq\left|X^{*}\right|-\operatorname{dim}_{K} C_{X^{*}}(d)+1 .
$$

The parameters of evaluation codes over finite fields have been computed in a number of cases. If $\mathbb{X}^{*}$ is the image of the affine space $K^{s}$ under the map $K^{s} \rightarrow \mathbb{P}^{s}, x \mapsto[(1, x)]$, the parameters of $C_{\mathbb{X}^{*}}(d)$ are described in [4, Theorem 2.6.2].

In this article we focus on linear codes parameterized by the edges of a graph $\mathcal{G}$ (see Definition 2.4) which has $m$ components and each component is an odd cycle; all our work is based on the affine space. In [16], the authors work with codes parameterized by even cycles over the projective space, they find an explicit description for a set of generators of the vanishing ideal (see Definition 1.1) associated to an even cycle. In the same article, we can also find the length of the code associated to a graph $\mathcal{G}$ with $m$ connected components (see [16, Theorem 3.2]). In [12], the authors work with codes parameterized by odd cycles over the projective space and they prove that parameterized sets by odd cycles over the projective space are projective torus. Therefore, if we work with codes parameterized by odd cycles over the projective space, we get parameterized codes over projective torus, and these codes are very well known. There is not any paper that works with parameterized codes by odd cycles over the affine space, in contrast with [12], we are going to see that if $2 \mid q-1$ then affine sets parameterized by odd cycles are not affine torus.

The contents of this paper are as follows. In Section 2 we introduce the preliminaries and explain the connection between the codes and graphs. In Section 3 we provide an explicit description of a set of generators from the vanishing ideal of an affine set parameterized by a graph $\mathcal{G}$ with $m$ connected components, where each component is an odd cycle (see Theorem 3.11). The set parameterized by the edges of a graph $\mathcal{G}$ will be denoted by $X_{\mathcal{G}}^{*}$ (see Definition 2.4) . 
Definition 1.1. (i) Let $X \subseteq K^{s}$. We set:

$$
\mathbf{I}(X)=\left\{f \in S \mid f\left(x_{1}, \ldots, x_{s}\right)=0 \forall x=\left(x_{1}, \ldots, x_{s}\right) \in X\right\} .
$$

(ii) Let $\mathbb{X} \subseteq \mathbb{P}_{K}^{s-1}$. We set:

$$
\mathbf{I}(\mathbb{X})=\langle\{f \in S \mid f \text { is homogeneous and } f(x)=0 \forall x \in \mathbb{X}\}\rangle
$$

Clearly $\mathbf{I}(X)$ is an ideal, we will call to $\mathbf{I}(X)$ (resp. $\mathbf{I}(\mathbb{X})$ ) the vanishing ideal of $X$ (resp. $\mathbb{X})$.

For a set $X^{*}$ parameterized by monomials, the main algebraic fact about $\mathbf{I}\left(X^{*}\right)$ that we use is a remarkable result of [15] showing that $\mathbf{I}\left(X^{*}\right)$ is a binomial ideal. In Section 4 we give an explicit formula for the regularity of $\mathbf{I}\left(X_{\mathcal{G}}^{*}\right)$, where $\mathcal{G}$ is graph with $m$ connected components and each component is an odd cycle. Finally, in Section 5, if $\mathcal{G}$ is an odd cycle of length $k$, we prove that the Hilbert function of $\mathbf{I}\left(X_{\mathcal{G}}^{*}\right)$ can be written as a linear combination of Hilbert functions of degenerate torus.

For all unexplained terminology and additional information we refer to [7] (for the theory of binomial ideals), [2, 19] (for the theory of polynomial ideals and Hilbert functions).

\section{Preliminaries: Codes Associated to a Graphs}

We will use the notation and definitions used in the introduction. In this section, we introduce the connection between graphs and codes and we present the basic theory of Hilbert functions that we will use later.

Theorem 2.1. (Combinatorial Nullstellensatz [1, Theorem 1.2]) Let $R=$ $K\left[y_{1}, \ldots, y_{n}\right]$ be a polynomial ring over a field $K$, let $f \in R$, and let $a=$ $\left(a_{1}, \ldots, a_{n}\right) \in \mathbb{N}^{n}$. Suppose that the coefficient of $y^{a}$ in $f$ is non zero and $\operatorname{deg}(f)=a_{1}+\cdots+a_{n}$. If $S_{1}, \ldots, S_{n}$ are subsets of $K$, with $\left|S_{i}\right|>a_{i}$ for all $i$, then there are $s_{1} \in S_{1}, \ldots, s_{n} \in S_{n}$ such that $f\left(s_{1}, \ldots, s_{n}\right) \neq 0$.

Let $X^{*} \subseteq K^{s-1}$ be an affine algebraic toric set parameterized by $y^{v_{1}}, \ldots$, $y^{v_{s-1}}$. The kernel of the evaluation map ev ev $_{d}$ defined in Eq. (2), is $\mathbf{I}\left(X^{*}\right)_{\leq d}$; in other words $\mathbf{I}\left(X^{*}\right) \leq d$ is the set of all the polynomials of degree less or equal to $d$ that are in $\mathbf{I}\left(X^{*}\right)$, thus there is an isomorphism of $K$-vector spaces:

$$
S_{\leq d} / \mathbf{I}\left(X^{*}\right)_{\leq d} \simeq C_{X^{*}}(d) .
$$

The affine Hilbert function of $\mathbf{I}\left(X^{*}\right)$ is given by: 


$$
H_{X^{*}}(d)=\operatorname{dim}_{K} S_{\leq d} / \mathbf{I}\left(X^{*}\right)_{\leq d}=\operatorname{dim}_{K} C_{X^{*}}(d) .
$$

Let $\mathbb{X} \subseteq \mathbb{P}_{K}^{s-1}$ be the projective closure of $X^{*}$ and let $C_{\mathbb{X}}(d)$ be a projective Reed-Muller code of degree $d$. It is shown that the codes $C_{\mathbb{X}}(d)$ and $C_{X^{*}}(d)$ have the same basic parameters (see [15, Theorem 2.4]). The kernel of the evaluation map ev ${ }_{d}$, defined in Eq. (1), is precisely $\mathbf{I}(\mathbb{X})_{d}$ the degree $d$ piece of $\mathbf{I}(\mathbb{X})$. Hence there is an isomorphism of $K$-vector spaces

$$
S_{d} / \mathbf{I}(\mathbb{X})_{d} \simeq C_{\mathbb{X}}(d) .
$$

Two of the basic parameters of $C_{\mathbb{X}}(d)$ can be expressed using Hilbert functions of standard graded algebras [19], as we now explain. Recall that the Hilbert function of $\mathbf{I}(\mathbb{X})$ is given by

$$
H_{\mathbb{X}}(d):=\operatorname{dim}_{K}(S / \mathbf{I}(\mathbb{X}))_{d}=\operatorname{dim}_{K} S_{d} / \mathbf{I}(\mathbb{X})_{d}=\operatorname{dim}_{K} C_{\mathbb{X}}(d) .
$$

The unique polynomial $h_{\mathbb{X}}(t)=\sum_{i=0}^{k-1} c_{i} t^{i} \in \mathbb{Z}[t]$ of degree $k-1=\operatorname{dim}(S / \mathbf{I}(\mathbb{X}))$ 1 such that $h_{\mathbb{X}}(d)=H_{\mathbb{X}}(d)$ for $d \gg 0$ is called the Hilbert polynomial of $\mathbf{I}(\mathbb{X})$. The integer $c_{k-1}(k-1)$ !, denoted by $\operatorname{deg}(S / \mathbf{I}(\mathbb{X}))$, is called the degree or multiplicity of $S / \mathbf{I}(\mathbb{X})$. In our situation $h_{\mathbb{X}}(t)$ is a non-zero constant because $S / \mathbf{I}(\mathbb{X})$ has dimension 1. Furthermore $h_{\mathbb{X}}(d)=|\mathbb{X}|$ for $d \geq|\mathbb{X}|-1$, see [13, Lecture 13]. This means that $|\mathbb{X}|$ equals the degree of $S / \mathbf{I}(\mathbb{X})$. Thus $H_{\mathbb{X}}(d)$ and $\operatorname{deg}(S / \mathbf{I}(\mathbb{X}))$ equal the dimension and the length of $C_{\mathbb{X}}(d)$ respectively. There are algebraic methods, based on elimination theory and Gröbner bases, to compute the dimension and the length of $C_{\mathbb{X}}(d)[17]$.

Definition 2.2. The regularity index of $S / \mathbf{I}(\mathbb{X})$, denoted by $\operatorname{reg}(S / \mathbf{I}(\mathbb{X}))$, is the least integer $p \geq 0$ such that $h_{\mathbb{X}}(d)=H_{\mathbb{X}}(d)$ for $d \geq p$.

As $S / \mathbf{I}(\mathbb{X})$ is a 1-dimensional Cohen-Macaulay graded algebra [8], the regularity index of $S / \mathbf{I}(\mathbb{X})$ is the Castelnuovo-Mumford regularity of $S / \mathbf{I}(\mathbb{X})[6]$. By Hilbert-Serre Theorem, the Hilbert series of $S / \mathbf{I}(\mathbb{X})$ can be uniquely written as $F_{\mathbb{X}}(t)=\frac{f(t)}{1-t}$, where $f$ is a polinomial of degree equal to the regularity of $S / \mathbf{I}(\mathbb{X})$. From the exact sequence:

$$
0 \rightarrow(S / \mathbf{I}(\mathbb{X}))[-1] \stackrel{t_{\text {s }}}{\rightarrow} S / \mathbf{I}(\mathbb{X}) \rightarrow S /\left(t_{s}, \mathbf{I}(\mathbb{X})\right) \rightarrow 0,
$$

we deduce $F\left(S /\left(t_{s}, \mathbf{I}(\mathbb{X})\right), t\right)=f(t)$, where $F\left(S /\left(t_{s}, \mathbf{I}(\mathbb{X})\right), t\right)$ is the Hilbert series of $S /\left(t_{s}, \mathbf{I}(\mathbb{X})\right)$.

Let $>$ be a monomial order on $S$ and let $\langle 0\rangle \neq I \subseteq S$ be an ideal. If $f$ is a non-zero polynomial in $S$, we can write:

$$
f=\lambda_{1} t^{\alpha_{1}}+\cdots+\lambda_{r} t^{\alpha_{r}},
$$


with $\lambda_{i} \in K^{*}$ for all $i$ and $t^{\alpha_{1}}>\cdots>t^{\alpha_{r}}$. The leading monomial $t^{\alpha_{1}}$ of $f$ is denoted by $L M(f)$ and the leading term $\lambda_{1} L M(f)$ of $f$ is denoted by $L T(f)$. We denote by $L T(I)$ the set of leading terms of elements of $I$. The ideal of leading terms of $I$ is the monomial ideal of $S$ given by:

$$
\langle L T(I)\rangle \text {. }
$$

Definition 2.3. Let $\langle 0\rangle \neq I \subseteq S$ be and ideal. A monomial $t^{a}$ is called a standard monomial of $S / I$, with respect to $>$, if $t^{a}$ is not the leading monomial of any polynomial in $I$, that is, $t^{a} \notin\langle L T(I)\rangle$. A polynomial $f$ is called standard if $f \neq 0$ and $f$ is $k$-linear combination of standard monomials.

The set of standard monomials, denoted by $\Delta_{>}(I)$, is called the footprint of $S / I$. The image of $\Delta_{>}(I)$, under the canonical map $S \longrightarrow S / I$, is a basis of $S / I$ as a $K$-vector space. In particular If $X^{*} \subseteq K^{s-1}$ is an affine algebraic toric set parameterized by $y^{v_{1}}, \ldots, y^{v_{s-1}}$, then $H_{X^{*}}(d)$ is the number of standard monomials of degree less or equal to $d$.

The affine algebraic toric set parameterized by $y_{1}, \ldots, y_{s}$ will be denoted by $T^{s}$. We call $T^{s}$ an affine torus;

$$
T^{s}=\left\{\left(x_{1}, \ldots, x_{s}\right) \mid x_{i} \in K^{*}\right\}
$$

It is known that $\mathbf{I}\left(T^{s}\right)=\left\langle\left\{t_{i}^{q-1}-1\right\}_{i=1}^{s}\right\rangle$. Let $X^{*} \subseteq K^{s}$ be an affine algebraic toric set parameterized by $y^{v_{1}}, \ldots, y^{v_{s}}$. By [15] we know that $\mathbf{I}\left(X^{*}\right)$ is generated by binomials $t^{a}-t^{b} \in S$ where $a, b \in \mathbb{N}^{s}$. In addition there are a few observations to be made.

- Since $X^{*} \subseteq T^{s}$, then $\mathbf{I}\left(T^{s}\right) \subseteq \mathbf{I}\left(X^{*}\right)$, hence $\left\{t_{i}^{q-1}-1\right\}_{i=1}^{s} \subseteq \mathbf{I}\left(X^{*}\right)$.

- Let $f=t^{a}-t^{b}$ be a nonzero binomial of $S$. If $\operatorname{gcd}\left(t^{a}, t^{b}\right) \neq 1$, then we can factor the greatest common divisor $t^{c}$ from both $t^{a}$ and $t^{b}$ to obtain $f=t^{c}\left(t^{a^{\prime}}-t^{b^{\prime}}\right)$, for some $a^{\prime}, b^{\prime} \in \mathbb{N}^{s}$. Since $t^{c}$ never is zero on $T^{s}$, for any $c \in \mathbb{N}^{s}$, we deduce that $f \in \mathbf{I}\left(X^{*}\right)$ if and only if $t^{a^{\prime}}-t^{b^{\prime}} \in \mathbf{I}\left(X^{*}\right)$. Accordingly, when looking for binomials generators of $\mathbf{I}\left(X^{*}\right)$ we may restrict ourselves to those binomials $t^{a}-t^{b}$ such that $t^{a}$ and $t^{b}$ have no common divisors.

- Given $a=\left(a_{1}, \ldots, a_{s}\right) \in \mathbb{N}^{s}$, we set $|a|=a_{1}+\cdots+a_{s}$ and $\operatorname{supp}(a)=\{i \mid$ $\left.a_{i} \neq 0\right\}$. Then clearly, $t^{a}$ and $t^{b}$ have no common divisors if and only if $\operatorname{supp}(a) \cap \operatorname{supp}(b)=\emptyset$. 
Let $\mathcal{G}$ be a simple graph with vertex set $V_{\mathcal{G}}=\left\{v_{1}, \ldots, v_{n}\right\}$ and edge set $E_{\mathcal{G}}=\left\{e_{1}, \ldots, e_{s}\right\}$. For and edge $e_{i}=\left\{v_{j}, v_{k}\right\}$, where $v_{j}, v_{k} \in V_{\mathcal{G}}$, let $\nu_{i}=$ $\mathbf{e}_{j}+\mathbf{e}_{k} \in \mathbb{N}^{n}$, where, for $1 \leq j \leq n, \mathbf{e}_{j}$ is the $j$-th element of the canonical basis of $\mathbb{Q}^{n}$.

Definition 2.4. The affine algebraic toric set associated to $\mathcal{G}$ is the affine toric set parameterized by the $n$-tuples $\nu_{1}, \ldots, \nu_{s} \in \mathbb{N}^{n}$, obtained from the edges of $\mathcal{G}$. If $X_{\mathcal{G}}^{*}$ is the affine parameterized toric set associated to $\mathcal{G}$ we call its associated linear code $C_{X_{9}^{*}}(d)$ the parameterized affine code associated to $\mathcal{G}$ and we refer to the vanishing ideal of $X_{\mathcal{G}}^{*}$ as the vanishing ideal over $\mathcal{G}$.

If $x=\left(x_{1}, \ldots, x_{n}\right) \in\left(K^{*}\right)^{n}$ and $e_{i}=\left\{v_{j}, v_{k}\right\}$ is an edge of $\mathcal{G}$, we set $x^{e_{i}}=x^{\mathbf{e}_{j}+\mathbf{e}_{k}}=x_{j} x_{k}$, so that the structural map $\theta:\left(K^{*}\right)^{n} \rightarrow X_{\mathcal{G}}^{*}$ is given by $x \rightarrow\left(x^{e_{1}}, \ldots, x^{e_{s}}\right)$. If $\mathcal{G}$ is a graph with $m$ connected components, of which $\epsilon$ are non-bipartite, the length of $C_{X_{S}^{*}}(d)$ has been determined.

Theorem 2.5. Let $\mathcal{G}$ be a graph with $m$ connected components, of which $\epsilon$ are non-bipartite. Suppose that $\mathcal{G}$ has $n$ vertices. Then:

$$
o\left(X_{\mathcal{G}}^{*}\right)= \begin{cases}(q-1)^{n-m+\epsilon} & \text { if } 2 \nmid q-1 \\ \frac{(q-1)^{n-m+\epsilon}}{2^{\epsilon}} & \text { if } 2 \mid q-1\end{cases}
$$

Proof. It follows from [16, Lemma 3.1].

Corollary 2.6. Let $\mathcal{G}$ be a graph with $m$ connected components. Suppose that each component is a $k$-cycle and $k$ is odd. Then:

$$
o\left(X_{\mathcal{G}}^{*}\right)= \begin{cases}(q-1)^{k m} & \text { if } 2 \nmid q-1 \\ \frac{(q-1)^{k m}}{2^{m}} & \text { if } 2 \mid q-1\end{cases}
$$

\section{Vanishing Ideals of Odd Cycles}

In this Section we give an explicit description of the vanishing ideal associated to a graph $\mathcal{G}$ with $m$ connected components and each component is an odd cycle.

We continue to use the notation and definitions used in the introduction. Let $\mathcal{G}$ be a graph with $m$ connected components, suppose that each component is a $k$-cycle with $k=2 \gamma+1$. Let $C_{1}^{k}=x_{1} \cdots x_{k} x_{1}, C_{2}^{k}=x_{k+1} \cdots x_{2 k} x_{k+1}, \ldots$, $C_{m-1}^{k}=x_{(m-2) k+1} \cdots x_{(m-1) k} x_{(m-2) k+1}, C_{m}^{k}=x_{(m-1) k+1} \cdots x_{m k} x_{(m-1) k+1}$ be the $m$ components of $\mathcal{G}$. If $2 \nmid q-1$, then $o\left(X_{\mathcal{G}}^{*}\right)=(q-1)^{k m}$, therefore $X_{\mathcal{G}}^{*}$ is an affine torus. Suppose that $2 \mid q-1$. Let: 


$$
\begin{gathered}
F_{1}=\{A \subseteq\{1, \ldots, k\}|| A \mid=\gamma\}, \\
F_{2}=\{A \subseteq\{k+1, \ldots, 2 k\}|| A \mid=\gamma\}, \\
\vdots \\
F_{m}=\{A \subseteq\{(m-1) k+1, \ldots, m k\}|| A \mid=\gamma\} .
\end{gathered}
$$

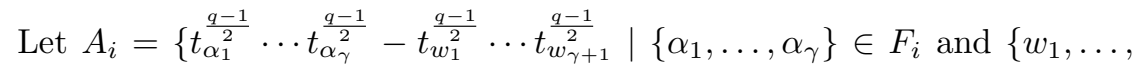
$\left.\left.w_{\gamma+1}\right\}=\{(i-1) k+1, \ldots, i k\}-\left\{\alpha_{1}, \ldots, \alpha_{\gamma}\right\}\right\}$. Note that $A_{i} \subseteq K\left[t_{(i-1) k+1}, \ldots\right.$, $\left.t_{i k}\right]$.

Lemma 3.1. For each $i \in\{1, \ldots, m\}$ we have that $A_{i} \subseteq \mathbf{I}\left(X_{\mathcal{G}}^{*}\right)$.

Proof. Let $i \in\{1, \ldots, m\}$ and $f \in A_{i}$. Let $f=t_{\alpha_{1}^{\frac{q-1}{2}}} \cdots t_{\alpha_{\gamma}}^{\frac{q-1}{2}}-t_{w_{1}^{2}}^{\frac{q-1}{2}} \cdots t_{w_{\gamma+1}}^{\frac{q-1}{2}}$ where $\left\{\alpha_{1}, \ldots, \alpha_{\gamma}\right\} \in F_{i}$ and $\left\{w_{1}, \ldots, w_{\gamma+1}\right\}=\{(i-1) k+1, \ldots, i k\}-\left\{\alpha_{1}, \ldots\right.$, $\left.\alpha_{\gamma}\right\}$.

Let $g=t_{(i-1) k+1}^{\frac{q-1}{2}} \cdots t_{(i-1) k+\gamma}^{\frac{q-1}{2}}-t_{(i-1) k+\gamma+1}^{\frac{q-1}{2}} \cdots t_{i k}^{\frac{q-1}{2}}$. For each $j \in\{1, \ldots, \gamma\}$, let:

$$
G_{j}\left(t_{(i-1) k+1}, \ldots, t_{i k}\right)=\left\{\begin{array}{cl}
1 & \text { if } \alpha_{j}=(i-1) k+j \\
t_{\alpha_{j}}^{\frac{q-1}{2}} t_{(i-1) k+j}^{\frac{q-1}{2}} & \text { if } \alpha_{j} \neq(i-1) k+j
\end{array}\right.
$$

Let $h=f G_{1} \cdots G_{\gamma}$. Let $w=\left(x_{(i-1) k+1} x_{(i-1) k+2}, \ldots, x_{i k-1} x_{i k}, x_{i k} x_{(i-1) k+1}\right)$ where $x_{i} \in K^{*}$. Then we obtain:

$$
h(w)=f(w) \eta=g(w),
$$

where $\eta \in K^{*}$. Therefore:

$$
\begin{gathered}
g(w)= \\
\left(x_{(i-1) k+1} x_{(i-1) k+2}\right)^{\frac{q-1}{2}}\left(x_{(i-1) k+2} x_{(i-1) k+3}\right)^{\frac{q-1}{2}} \cdots\left(x_{(i-1) k+\gamma} x_{(i-1) k+\gamma+1}\right)^{\frac{q-1}{2}}- \\
\left(x_{(i-1) k+\gamma+1} x_{(i-1) k+\gamma+2}\right)^{\frac{q-1}{2}} \cdots\left(x_{i k} x_{(i-1) k+1}\right)^{\frac{q-1}{2}} .
\end{gathered}
$$

Note that $g(w)=x_{(i-1) k+1}^{\frac{q-1}{2}} x_{(i-1) k+\gamma+1}^{\frac{q-1}{2}}-x_{(i-1) k+\gamma+1}^{\frac{q-1}{2}} x_{(i-1) k+1}^{\frac{q-1}{2}}$, then $g(w)=$ 0 . It follows that $f(w)=0$, therefore $f \in \mathbf{I}\left(X_{\mathcal{G}}^{*}\right)$.

Proposition 3.2. Let $f=t^{a}-t^{b}$ be a binomial in $K\left[t_{1}, \ldots, t_{k m}\right]$ with $\operatorname{supp}(a) \cap$ $\operatorname{supp}(b)=\emptyset$. Then $f=g+f^{\prime}$ where $g \in \mathbf{I}\left(T^{k m}\right)$ and $f^{\prime}=t^{a^{\prime}}-t^{b^{\prime}}$ is a binomial such that none of its two terms is divisible by any $t_{i}^{q-1}$ for all $i$ and $\operatorname{supp}\left(a^{\prime}\right) \cap \operatorname{supp}\left(b^{\prime}\right)=\emptyset$. 
Proof. If $t^{a}$ and $t^{b}$ are not divisible by any $t_{i}^{q-1}$ for all $i$, we can take $g=0$ and $f=f^{\prime}$. Then we can write $f$ as:

$$
f=m_{1} t_{\alpha_{1}}^{a_{1}} \cdots t_{\alpha_{n}}^{a_{n}}-m_{2} t_{w_{1}}^{b_{1}} \cdots t_{w_{r}}^{b_{r}},
$$

where $t^{a}=m_{1} t_{\alpha_{1}}^{a_{1}} \cdots t_{\alpha_{n}}^{a_{n}}$ and $t^{b}=m_{2} t_{w_{1}}^{b_{1}} \cdots t_{w_{r}}^{b_{r}} \cdot m_{1}$ and $m_{2}$ are monomials such that none of them is divisible by any $t_{i}^{q-1}$ for all $i . m_{1}$ is not divisible by any $t_{\alpha_{i}}$ and $a_{i} \geq q-1$ for all $i . m_{2}$ is not divisible by any $t_{w_{j}}$ and $b_{j} \geq q-1$ for all $j$.

By the division algorithm we can write $a_{i}=(q-1) q_{i}+r_{i}$ where $0 \leq r_{i}<$ $q-1$ for all $i=1, \ldots, n$. Note that $q_{i}>0$ for all $i$. Then $m_{1} t_{\alpha_{1}}^{a_{1}} \cdots t_{\alpha_{n}}^{a_{n}}=$ $t_{\alpha_{1}}^{r_{1}} m_{1}\left(t_{\alpha_{1}}^{(q-1)\left(q_{1}-1\right)} t_{\alpha_{2}}^{a_{2}} \cdots t_{\alpha_{n}}^{a_{n}}\right)\left(t_{\alpha_{1}}^{q-1}-1\right)+t_{\alpha_{1}}^{r_{1}} m_{1}\left(t_{\alpha_{1}}^{(q-1)\left(q_{1}-1\right)} \cdots t_{\alpha_{n}}^{a_{n}}\right)$. Let $m_{1}^{\prime}=$ $m_{1} t_{\alpha_{1}}^{a_{1}} \cdots t_{\alpha_{n}}^{a_{n}}$, then:

$$
m_{1}^{\prime}=\left(\sum_{j=1}^{q_{1}} t_{\alpha_{1}}^{(q-1)\left(q_{1}-j\right)}\right) t_{\alpha_{1}}^{r_{1}} m_{1}\left(t_{\alpha_{2}}^{a_{2}} \cdots t_{\alpha_{n}}^{a_{n}}\right)\left(t_{\alpha_{1}}^{q-1}-1\right)+t_{\alpha_{1}}^{r_{1}} m_{1}\left(t_{\alpha_{2}}^{a_{2}} \cdots t_{\alpha_{n}}^{a_{n}}\right) .
$$

If we do the previous analysis with the term $t_{\alpha_{1}}^{r_{1}} m_{1}\left(t_{\alpha_{2}}^{a_{2}} \cdots t_{\alpha_{n}}^{a_{n}}\right)$ and we continue, we get:

$$
m_{1}^{\prime}=g_{1}+\left(t_{\alpha_{1}}^{r_{1}} t_{\alpha_{2}}^{r_{2}} \cdots t_{\alpha_{n}}^{r_{n}}\right) m_{1}
$$

where $g_{1} \in \mathbf{I}\left(T^{k m}\right)$. Let $m_{2}^{\prime}=m_{2} t_{w_{1}}^{b_{1}} \cdots t_{w_{r}}^{b_{r}}$. By the division algorithm we can write $b_{j}=(q-1) q_{j}^{\prime}+b_{j}^{\prime}$ where $0 \leq b_{j}^{\prime}<q-1$ for all $j=1, \ldots, r$. Note that $q_{j}^{\prime}>0$ for all $j$. If we do with $m_{2}^{\prime}$ the same procedure that we did with $m_{1}^{\prime}$, we get:

$$
m_{2}^{\prime}=g_{2}+\left(t_{w_{1}}^{b_{1}^{\prime}} t_{w_{2}}^{b_{2}^{\prime}} \cdots t_{w_{r}}^{b_{r}^{\prime}}\right) m_{2}
$$

where $g_{2} \in \mathbf{I}\left(T^{k m}\right)$. Then $f=g_{1}-g_{2}+\left(t_{\alpha_{1}}^{r_{1}} t_{\alpha_{2}}^{r_{2}} \cdots t_{\alpha_{n}}^{r_{n}}\right) m_{1}-\left(t_{w_{1}}^{b_{1}^{\prime}} t_{w_{2}}^{b_{2}^{\prime}} \cdots t_{w_{r}}^{b_{r}^{\prime}}\right) m_{2}$. Let $g=g_{1}-g_{2}$ and $f^{\prime}=\left(t_{\alpha_{1}}^{r_{1}} t_{\alpha_{2}}^{r_{2}} \cdots t_{\alpha_{n}}^{r_{n}}\right) m_{1}-\left(t_{w_{1}}^{b_{1}^{\prime}} t_{w_{2}}^{b_{2}^{\prime}} \cdots t_{w_{r}}^{b_{r}^{\prime}}\right) m_{2}$.

Remark 3.3. As a consequence of the last proposition we get $f \in \mathbf{I}\left(X_{\mathcal{G}}^{*}\right)$ if and only if $f^{\prime} \in \mathbf{I}\left(X_{\mathcal{G}}^{*}\right)$.

Proposition 3.4. Let $i \in\{1, \ldots, m\}$. Let $f=t^{a}-t^{b} \in K\left[t_{(i-1) k+1}, \ldots, t_{i k}\right]$ with $\operatorname{supp}(a) \cap \operatorname{supp}(b)=\emptyset$. Suppose that:

- All the coordinates of $a$ and $b$ are equal to $\frac{q-1}{2}$.

- $a$ and b have no empty support. 
- $\operatorname{supp}(a) \cup \operatorname{supp}(b)=\{(i-1) k+1, \ldots, i k\}$.

Then we obtain:

$$
f \in\left\langle\left\{t_{i}^{q-1}-1\right\}_{i=1}^{k m} \cup\left(\bigcup_{i=1}^{m} A_{i}\right)\right\rangle
$$

Proof. Let $J=\left\langle\left\{t_{i}^{q-1}-1\right\}_{i=1}^{k m} \cup\left(\bigcup_{i=1}^{m} A_{i}\right)\right\rangle$. We can write $f$ as:

$$
f=t_{a_{1}^{2}}^{\frac{q-1}{2}} \cdots t_{a_{n}^{2}}^{\frac{q-1}{2}}-t_{b_{1}}^{\frac{q-1}{2}} \cdots t_{b_{r}}^{\frac{q-1}{2}}
$$

where $t^{a}=t_{a_{1}}^{\frac{q-1}{2}} \cdots t_{a_{n}}^{\frac{q-1}{2}}$ and $t^{b}=t_{b_{1}}^{\frac{q-1}{2}} \cdots t_{b_{r}}^{\frac{q-1}{2}}$. By hypothesis we have that $\operatorname{supp}(a) \cup \operatorname{supp}(b)=\{(i-1) k+1, \ldots, i k\}$, therefore $n+r=k$. Suppose that $r>\gamma$. Let $r=\gamma+\beta$. On the other hand we have:

$$
-t_{b_{\gamma+1}}^{\frac{q-1}{2}} \cdots t_{b_{\gamma+\beta}}^{\frac{q-1}{2}}\left[t_{b_{1}}^{\frac{q-1}{2}} \cdots t_{b_{\gamma}}^{\frac{q-1}{2}}-\left(t_{b_{\gamma+1}}^{\frac{q-1}{2}} \cdots t_{b_{\gamma+\beta}}^{\frac{q-1}{2}}\right) t^{a}\right]=-t^{b}+t^{a}\left(t_{b_{\gamma+1}}^{q-1} \cdots t_{b_{\gamma+\beta}}^{q-1}\right)
$$

note that $\beta+n=\gamma+1$, therefore $t_{b_{1}}^{\frac{q-1}{2}} \cdots t_{b_{\gamma}}^{\frac{q-1}{2}}-\left(t_{b_{\gamma+1}}^{\frac{q-1}{2}} \cdots t_{b_{\gamma+\beta}}^{\frac{q-1}{2}}\right) t^{a} \in J$. We can write $m^{\prime}=t^{a}\left(t_{b_{\gamma+1}}^{q-1} \cdots t_{b_{\gamma+\beta}}^{q-1}\right)$ as $m^{\prime}=t^{a}\left(t_{b_{\gamma+2}}^{q-1} \cdots t_{b_{\gamma+\beta}}^{q-1}\right)\left[t_{b_{\gamma+1}}^{q-1}-1\right]+$ $t^{a}\left(t_{b_{\gamma+2}}^{q-1} \cdots t_{b_{\gamma+\beta}}^{q-1}\right)$. If we do the same with the term $t^{a}\left(t_{b_{\gamma+2}}^{q-1} \cdots t_{b_{\gamma+\beta}}^{q-1}\right)$ and we continue, we get:

$$
m^{\prime}=g+t^{a}
$$

where $g \in \mathbf{I}\left(T^{k m}\right)$. Then $-t_{b_{\gamma+1}}^{\frac{q-1}{2}} \cdots t_{b_{\gamma+\beta}}^{\frac{q-1}{2}}\left[t_{b_{1}}^{\frac{q-1}{2}} \cdots t_{b_{\gamma}}^{\frac{q-1}{2}}-\left(t_{b_{\gamma+1}}^{\frac{q-1}{2}} \cdots t_{b_{\gamma+\beta}}^{\frac{q-1}{2}}\right) t^{a}\right]=$ $-t^{b}+g+t^{a}$, therefore $f=-t_{b_{\gamma+1}}^{\frac{q-1}{2}} \cdots t_{b_{\gamma+\beta}}^{\frac{q-1}{2}}\left[t_{b_{1}}^{\frac{q-1}{2}} \cdots t_{b_{\gamma}}^{\frac{q-1}{2}}-\left(t_{b_{\gamma+1}}^{\frac{q-1}{2}} \cdots t_{b_{\gamma+\beta}}^{\frac{q-1}{2}}\right) t^{a}\right]-g$. It follows that $f \in J$. If $r \leq \gamma$, it is easy to see that $n>\gamma$, then we use the same proof as above.

Lemma 3.5. Let $f \in \mathbf{I}\left(X_{\mathcal{G}}^{*}\right)$, then $f\left(a_{1}^{2}, \ldots, a_{k m}^{2}\right)=0$ for all $a=\left(a_{1}, \ldots, a_{k m}\right) \in$ $\left(\mathbb{F}_{q}^{*}\right)^{k m}$.

Proof. Let $i \in\{0,1, \ldots, m-1\}$ and $\bar{a}=\left(a_{i k+1}^{2}, a_{i k+2}^{2}, \ldots, a_{k(i+1)}^{2}\right)$. Let $\alpha_{j}=$ $a_{i k+j}^{2}$ for $j=1, \ldots, k$. Let:

$$
\begin{gathered}
x_{i k+2}=\alpha_{1} x_{i k+1}^{-1}, \\
x_{i k+3}=\alpha_{2} \alpha_{1}^{-1} x_{i k+1}, \\
x_{i k+4}=\alpha_{3} \alpha_{2}^{-1} \alpha_{1} x_{i k+1}^{-1},
\end{gathered}
$$




$$
x_{i k+k}=\alpha_{k-1} \alpha_{k-2}^{-1} \cdots \alpha_{1} x_{i k+1},
$$

where $x_{i k+1}=a_{i k+k} a_{i k+k-1}^{-1} \cdots a_{i k+1}$. It is easy to see that $\bar{a}=\left(x_{i k+1} x_{i k+2}\right.$ $\left., x_{i k+2} x_{i k+3}, \ldots, x_{i k+k-1} x_{i k+k}, x_{i k+k} x_{i k+1}\right)$. It follows that $\left(a_{1}^{2}, \ldots, a_{k m}^{2}\right) \in$ $X_{\mathcal{G}}^{*}$, then $f\left(a_{1}^{2}, \ldots, a_{k m}^{2}\right)=0$.

Lemma 3.6. Let $i \in\{1, \ldots, m\}$ and $f=t^{a}-t^{b} \in \mathbf{I}\left(X_{C_{i}^{k}}^{*}\right) \backslash\{0\}$ with $\operatorname{supp}(a) \cap$ $\operatorname{supp}(b)=\emptyset$. Suppose that $\operatorname{deg}(f)_{t_{i}}<q-1$ for all $i$ and $\emptyset \neq \operatorname{supp}(a) \subsetneq$ $\{(i-1) k+1, \ldots, i k\}$. Then $\operatorname{supp}(b) \neq \emptyset$.

Proof. Suppose that $\operatorname{supp}(b)=\emptyset$. Then we get that $f=t^{a}-1$. Let $n=$ $|\operatorname{supp}(a)|$. We can write $f$ as:

$$
f\left(t_{\alpha_{1}}, \ldots, t_{\alpha_{n}}\right)=t_{\alpha_{1}}^{a_{1}} \cdots t_{\alpha_{n}}^{a_{n}}-1
$$

where $t^{a}=t_{\alpha_{1}}^{a_{1}} \cdots t_{\alpha_{n}}^{a_{n}}$. First we will prove that $a_{i}=\frac{q-1}{2}$ for all $i$. Let $\beta \in \mathbb{F}_{q}^{*}$. By the last lemma we get that $f\left(\beta^{2}, 1, \ldots, 1\right)=\left(\beta^{2}\right)^{a_{1}}-1=0$. Let $h\left(t_{\alpha_{1}}\right)=t_{\alpha_{1}}^{a_{1}}-1$, Let $S=\left\{\alpha^{2} \mid \alpha \in \mathbb{F}_{q}^{*}\right\}$. It is easy to see that $|S|=\frac{q-1}{2}$, then $h$ vanishes on $S$. Now we are going to examine the following cases:

- Suppose that $a_{1}<\frac{q-1}{2}$. By Combinatorial Nullstellensatz we get that $h=0$ and this is a contradiction because $a_{1}>0$.

- Suppose that $a_{1}>\frac{q-1}{2}$. Let $a_{1}=\frac{q-1}{2}+i_{1}$ where $0<i_{1}<\frac{q-1}{2}$. Let $h^{\prime}\left(t_{\alpha_{1}}\right)=t_{\alpha_{1}}^{i_{1}}-1$, it is clear that $h^{\prime}=h$ over $S$, then $h^{\prime}$ vanishes on $S$, by Combinatorial Nullstellensatz we get that $h^{\prime}=0$ and this is a contradiction.

Therefore the only possible case is when $a_{1}=\frac{q-1}{2}$. In this way we can prove that $a_{i}=\frac{q-1}{2}$ for all $i$. Let $\alpha \in \mathbb{F}_{q}^{*} \backslash S$. Now we are going to examine the following cases:

1) Suppose that $(i-1) k+1 \in \operatorname{supp}(a)$. If $(i-1) k+2 \notin \operatorname{supp}(a)$, then $(\alpha, \alpha, 1, \ldots, 1) \in X_{C_{i}^{k}}^{*}$, therefore $f(\alpha, \alpha, 1, \ldots, 1)=(\alpha)^{\frac{q-1}{2}}-1=0$ and this is a contradiction because $\alpha \notin S$. Suppose that $(i-1) k+2 \in$ $\operatorname{supp}(a)$, if $(i-1) k+3 \notin \operatorname{supp}(a)$, then $(1, \alpha, \alpha, 1, \ldots, 1) \in X_{C_{i}^{k}}^{*}$, thus $f(1, \alpha, \alpha, 1, \ldots, 1)=(\alpha)^{\frac{q-1}{2}}-1=0$, and this is a contradiction. If we continue, we get that $(i-1) k+1, \ldots, i k-1 \in \operatorname{supp}(a)$, then $i k \notin \operatorname{supp}(a)$ because $\operatorname{supp}(\mathrm{a}) \subsetneq\{(i-1) k+1, \ldots, i k\}$, then $(1, \ldots, 1, \alpha, \alpha) \in X_{C_{i}^{k}}^{*}$, and $f(1, \ldots, 1, \alpha, \alpha)=(\alpha)^{\frac{q-1}{2}}-1=0$ which is a contradiction. 
2) Suppose that $(i-1) k+1 \notin \operatorname{supp}(a)$. If $(i-1) k+2 \in \operatorname{supp}(a)$, then $(\alpha, \alpha, 1, \ldots, 1) \in X_{C_{i}^{k}}^{*}$, then $f(\alpha, \alpha, 1, \ldots, 1)=(\alpha)^{\frac{q-1}{2}}-1=0$ and this is a contradiction because $\alpha \notin S$. If we continue as in case 1 ), we get a contradiction.

It follows that $\operatorname{supp}(b) \neq \emptyset$.

Remark 3.7. Let $i \in\{1, \ldots, m\}$ and $f=t^{a}-t^{b} \in \mathbf{I}\left(X_{C_{i}^{k}}^{*}\right) \backslash\{0\}$ with $\operatorname{supp}(a) \cap \operatorname{supp}(b)=\emptyset$. Suppose that $\operatorname{deg}(f)_{t_{i}}<q-1$ for all $i$. If $\operatorname{supp}(a)=$ $\{(i-1) k+1, \ldots, i k\}$, then $\operatorname{supp}(b)=\emptyset$. If we follow the proof of the last lemma, we can prove that all the coordinates of $a$ are equal to $\frac{q-1}{2}$. Therefore:

$$
f=t_{(i-1) k+1}^{\frac{q-1}{2}} \cdots t_{i k}^{\frac{q-1}{2}}-1
$$

Let $h=t_{(i-1) k+1}^{\frac{q-1}{2}} \cdots t_{i k}^{\frac{q-1}{2}}$ and $h^{\prime}=t_{(i-1) k+1}^{\frac{q-1}{2}} \cdots t_{(1-1) k+\gamma}^{\frac{q-1}{2}}-t_{(i-1) k+\gamma+1}^{\frac{q-1}{2}} \cdots$ $t_{i k}^{\frac{q-1}{2}} . h^{\prime}$ belongs to $J=\left\langle\left\{t_{i}^{q-1}-1\right\}_{i=1}^{k m} \cup\left(\bigcup_{i=1}^{m} A_{i}\right)\right\rangle$. On the other hand we have:

$$
t_{(i-1) k+\gamma+1}^{\frac{q-1}{2}} \cdots t_{i k}^{\frac{q-1}{2}} h^{\prime}=h-t_{(i-1) k+\gamma+1}^{q-1} \cdots t_{i k}^{q-1} .
$$

Note that $t_{(i-1) k+\gamma+1}^{q-1} \cdots t_{i k}^{q-1}=t_{(i-1) k+\gamma+2}^{q-1} \cdots t_{i k}^{q-1}\left(t_{(i-1) k+\gamma+1}^{q-1}-1\right)+$ $t_{(i-1) k+\gamma+2}^{q-1} \cdots t_{i k}^{q-1}$. If we do the same with the term $t_{(i-1) k+\gamma+2}^{q-1} \cdots t_{i k}^{q-1}$ and we continue, we obtain:

$$
t_{(i-1) k+\gamma+1}^{q-1} \cdots t_{i k}^{q-1}=g+1
$$

where $g \in \mathbf{I}\left(T^{k m}\right)$. Therefore $t_{(i-1) k+\gamma+1}^{\frac{q-1}{2}} \cdots t_{i k}^{\frac{q-1}{2}} h^{\prime}=h-(g+1)=f-g$, then $f=t_{(i-1) k+\gamma+1}^{\frac{q-1}{2}} \cdots t_{i k}^{\frac{q-1}{2}} h^{\prime}+g$. It follows that $f \in J$.

Proposition 3.8. Let $i \in\{1, \ldots, m\}$ and $f=t^{a}-t^{b} \in \mathbf{I}\left(X_{C_{i}^{k}}^{*}\right) \backslash\{0\}$ with $\operatorname{supp}(a) \cap \operatorname{supp}(b)=\emptyset$. Suppose that $\operatorname{deg}(f)_{t_{i}}<q-1$ for all $i$ and $\emptyset \neq$ $\operatorname{supp}(a) \subsetneq\{(i-1) k+1, \ldots, i k\}$. Then all the coordinates of $a$ and $b$ are equal to $\frac{q-1}{2}$.

Proof. By hypothesis we have that $\operatorname{supp}(a) \neq \emptyset$, let $n=|\operatorname{supp}(a)|$. As $\operatorname{supp}(a) \subsetneq\{(i-1) k+1, \ldots, i k\}$, then $\operatorname{supp}(b) \neq \emptyset$, let $r=|\operatorname{supp}(b)|$. We can write $f$ as:

$$
f\left(t_{\alpha_{1}}, \ldots, t_{\alpha_{n}}, t_{w_{1}}, \ldots, t_{w_{r}}\right)=t_{\alpha_{1}}^{a_{1}} \cdots t_{\alpha_{n}}^{a_{n}}-t_{w_{1}}^{b_{1}} \cdots t_{w_{r}}^{b_{r}},
$$


where $t^{a}=t_{\alpha_{1}}^{a_{1}} \cdots t_{\alpha_{n}}^{a_{n}}$ and $t^{b}=t_{w_{1}}^{b_{1}} \cdots t_{\alpha_{r}}^{b_{r}}$. Let $(\beta, \mu) \in\left(\mathbb{F}_{q}^{*}\right)^{2}$. By Lemma 3.5 we get that $f\left(\beta^{2}, 1, \ldots, 1, \mu^{2}, 1, \ldots, 1\right)=\left(\beta^{2}\right)^{a_{1}}-\left(\mu^{2}\right)^{w_{1}}=0$. Let $h\left(t_{\alpha_{1}}, t_{w_{1}}\right)=$ $t_{\alpha_{1}}^{a_{1}}-t_{w_{1}}^{b_{1}}$, Let $S=\left\{\alpha^{2} \mid \alpha \in \mathbb{F}_{q}^{*}\right\}$. It is easy to see that $|S|=\frac{q-1}{2}$, then $h$ vanishes on $S^{2}$. Now we are going to examine the following cases:

- Suppose that $a_{1}, b_{1}<\frac{q-1}{2}$. By Combinatorial Nullstellensatz we get that $h=0$ and this is a contradiction because $a_{1}, b_{1}>0$.

- Suppose that $b_{1}<\frac{q-1}{2}$ and $a_{1}>\frac{q-1}{2}$. Let $a_{1}=\frac{q-1}{2}+i_{1}$ where $0<i_{1}<$ $\frac{q-1}{2}$. Let $h^{\prime}\left(t_{\alpha_{1}}, t_{w_{1}}\right)=t_{\alpha_{1}}^{i_{1}}-t_{w_{1}}^{b_{1}}$, it is clear that $h^{\prime}=h$ over $S^{2}$, then $h^{\prime}$ vanishes on $S^{2}$, by Combinatorial Nullstellensatz we get that $h^{\prime}=0$ and this is a contradiction. It is very similar the case $a_{1}<\frac{q-1}{2}$ and $b_{1}>\frac{q-1}{2}$.

- Suppose that $a_{1}, b_{1}>\frac{q-1}{2}$. Let $a_{1}=\frac{q-1}{2}+i_{1}$ and $b_{1}=\frac{q-1}{2}+i_{2}$ where $0<i_{1}, i_{2}<\frac{q-1}{2}$. Let $h^{\prime}\left(t_{\alpha_{1}}, t_{w_{1}}\right)=t_{\alpha_{1}}^{i_{1}}-t_{w_{1}}^{i_{2}}$, it is clear that $h^{\prime}=h$ over $S^{2}$, then $h^{\prime}$ vanishes on $S^{2}$, by Combinatorial Nullstellensatz we get that $h^{\prime}=0$ and this is a contradiction.

- Suppose that $b_{1}<\frac{q-1}{2}$ and $a_{1}=\frac{q-1}{2}$. Then $h\left(t_{\alpha_{1}}, t_{w_{1}}\right)=t_{\alpha_{1}}^{\frac{q-1}{2}}-t_{w_{1}}^{b_{1}}$. Let $h^{\prime}\left(t_{w_{1}}\right)=1-t_{w_{1}}^{b_{1}}$, it is clear that $h^{\prime}=h$ over $S^{2}$, then $h^{\prime}$ vanishes on $S$, by Combinatorial Nullstellensatz we get that $h^{\prime}=0$ and this is a contradiction because $b_{1}>0$. It is very similar the case $0<a_{1}<\frac{q-1}{2}$ and $b_{1}=\frac{q-1}{2}$.

As a result, the only possible case is when $b_{1}=a_{1}=\frac{q-1}{2}$. In this way we can prove that $a_{i}=\frac{q-1}{2}$ for all $i$ and $b_{j}=\frac{q-1}{2}$ for all $j$.

Proposition 3.9. Let $i \in\{1, \ldots, m\}$ and $f=t^{a}-t^{b} \in \mathbf{I}\left(X_{C_{i}^{k}}^{*}\right) \backslash\{0\}$ with $\operatorname{supp}(a) \cap \operatorname{supp}(b)=\emptyset$. Suppose that $\operatorname{deg}(f)_{t_{i}}<q-1$ for all $i$. Then $\operatorname{supp}(a) \cup$ $\operatorname{supp}(b)=\{(i-1) k+1, \ldots, i k\}$.

Proof. As $f$ is a nonzero polynomial then $\operatorname{supp}(a) \neq \emptyset$ or $\operatorname{supp}(b) \neq \emptyset$, Suppose that $\operatorname{supp}(a) \neq \emptyset$. If $\operatorname{supp}(a)=\{(i-1) k+1, \ldots, i k\}$, then $\operatorname{supp}(b)=\emptyset$, it is clear that $\operatorname{supp}(a) \cup \operatorname{supp}(b)=\{(i-1) k+1, \ldots, i k\}$. Suppose that $\operatorname{supp}(a) \subsetneq\{(i-1) k+1, \ldots, i k\}$, then we know that $\operatorname{supp}(b) \neq \emptyset$ and all the coordinates of $a$ and $b$ are equal to $\frac{q-1}{2}$. Suppose that $H=\operatorname{supp}(a) \cup \operatorname{supp}(b) \subsetneq$ $\{(i-1) k+1, \ldots, i k\}$. Let $S=\left\{\beta^{2} \mid \beta \in K^{*}\right\}$ and $\alpha \in K^{*} \backslash S$. Now we are going to examine the following cases:

1) Suppose that $(i-1) k+1 \in H$. If $(i-1) k+2 \notin H$, then $(\alpha, \alpha, 1, \ldots, 1) \in$ $X_{C_{i}^{k}}^{*}$, therefore $f(\alpha, \alpha, 1, \ldots, 1)=0$. Then we show $(\alpha)^{\frac{q-1}{2}}=1$ and this 
is a contradiction because $\alpha \notin S$. Suppose that $(i-1) k+2 \in H$, if $(i-$ $1) k+3 \notin H$, then $(1, \alpha, \alpha, 1, \ldots, 1) \in X_{C_{i}^{k}}^{*}$, therefore $f(1, \alpha, \alpha, 1, \ldots, 1)=$ 0 , then we find $(\alpha)^{\frac{q-1}{2}}=1$ and this is a contradiction. If we continue we get that $(i-1) k+1, \ldots, i k-1 \in H$, then $i k \notin H$ because $H \subsetneq\{(i-$ $1) k+1, \ldots, i k\}$, then $(1, \ldots, 1, \alpha, \alpha) \in X_{C_{i}^{k}}^{*}$, and $f(1, \ldots, 1, \alpha, \alpha)=0$ which is a contradiction.

2) Suppose that $(i-1) k+1 \notin H$. If $(i-1) k+2 \in H$, then $(\alpha, \alpha, 1, \ldots, 1) \in$ $X_{C_{i}^{k}}^{*}$, hence $f(\alpha, \alpha, 1, \ldots, 1)=0$, then we obtain $(\alpha)^{\frac{q-1}{2}}=1$ and this is a contradiction because $\alpha \notin S$. If we continue as in case 1 ), we get a contradiction.

Then $H=\{(i-1) k+1, \ldots, i k\}$. The proof is very similar if we suppose that $\operatorname{supp}(b) \neq \emptyset$.

Theorem 3.10. Let $\mathcal{G}$ be a graph with $m$ connected components, suppose that each component is a $k$-cycle with $k=2 \gamma+1$. The vanishing ideal of $X_{\mathcal{G}}^{*}$ is given by:

$$
\mathbf{I}\left(X_{\mathcal{G}}^{*}\right)=\left\langle\left\{t_{i}^{q-1}-1\right\}_{i=1}^{k m} \cup\left(\bigcup_{i=1}^{m} A_{i}\right)\right\rangle .
$$

Proof. Let $J=\left\langle\left\{t_{i}^{q-1}-1\right\}_{i=1}^{k m} \cup\left(\bigcup_{i=1}^{m} A_{i}\right)\right\rangle$. By Lemma 3.1 we get that $J \subseteq \mathbf{I}\left(X_{\mathcal{G}}^{*}\right)$. Now we will prove the other inclusion. We know that $\mathbf{I}\left(X_{\mathcal{G}}^{*}\right)$ is generated by binomials, let $f=t^{a}-t^{b} \in \mathbf{I}\left(X_{\mathcal{G}}^{*}\right)$ be a binomial; we can suppose that $\operatorname{supp}(a) \cap \operatorname{supp}(b)=\emptyset$. By Proposition 3.2 we can write $f$ as:

$$
f=g+f^{\prime},
$$

where $g \in \mathbf{I}\left(T^{k m}\right)$ and $f^{\prime}=t^{a^{\prime}}-t^{b^{\prime}}$ is a binomial such that none of its terms is divisible by any $t_{i}^{q-1}$ for all $i$ and $\operatorname{supp}\left(a^{\prime}\right) \cap \operatorname{supp}\left(b^{\prime}\right)=\emptyset$. Therefore $f \in J$ if and only if $f^{\prime} \in J$. We are going to prove that $f^{\prime} \in J$. Let:

$$
f^{\prime}=t^{a_{1}^{\prime}} \cdots t^{a_{m}^{\prime}}-t^{b_{1}^{\prime}} \cdots t^{b_{m}^{\prime}},
$$

where $t^{a^{\prime}}=t^{a_{1}^{\prime}} \cdots t^{a_{m}^{\prime}}$ and $t^{b^{\prime}}=t^{b_{1}^{\prime}} \cdots t^{b_{m}^{\prime}}$. $t^{a_{i}^{\prime}}$ and $t^{b_{i}^{\prime}}$ are monomials in $K\left[t_{(i-1) k+1}, \ldots, t_{i k}\right]$ for all $i=1, \ldots, m$. Let $i \in\{(i-1) k+1, \ldots, i k\}$. We can write $f^{\prime}$ as: 


$$
\begin{gathered}
f^{\prime}=\left(t^{a_{i}^{\prime}}-t^{b_{i}^{\prime}}\right) t^{a_{1}^{\prime}} \cdots t^{a_{i-1}^{\prime}} t^{a_{i+1}^{\prime}} \cdots t^{a_{m}^{\prime}}+t^{b_{i}^{\prime}} \\
{\left[t^{a_{1}^{\prime}} \cdots t^{a_{i-1}^{\prime}} t^{a_{i+1}^{\prime}} \cdots t^{a_{m}^{\prime}}-t^{b_{1}^{\prime}} \cdots t^{b_{i-1}^{\prime}} t^{b_{i+1}^{\prime}} \cdots t^{b_{m}^{\prime}}\right] .}
\end{gathered}
$$

As $f \in \mathbf{I}\left(X_{\mathcal{G}}^{*}\right)$, it follows that $f^{\prime} \in \mathbf{I}\left(X_{\mathcal{G}}^{*}\right)$. Let $\left(x_{(i-1) k+1}, \ldots, x_{i k}\right) \in$ $\left(\mathbb{F}_{q}^{*}\right)^{k}$, then $x=\left(1, \ldots, 1, x_{(i-1) k+1} x_{(i-1) k+2}, x_{(i-1) k+2} x_{(i-1) k+3}, \ldots, x_{i k-1} x_{i k}\right.$, $\left.x_{i k} x_{(i-1) k+1}, 1, \ldots, 1\right) \in X_{\mathcal{G}}^{*}$. Let $f_{i}=t^{a_{i}^{\prime}}-t^{b_{i}^{\prime}}$. As $f^{\prime}$ vanishes on $x$ we get that $f_{i}$ vanishes on $\left(x_{(i-1) k+1} x_{(i-1) k+2}, x_{(i-1) k+2} x_{(i-1) k+3}, \ldots, x_{i k-1} x_{i k}\right.$, $\left.x_{i k} x_{(i-1) k+1}\right)$, then $f_{i} \in \mathbf{I}\left(X_{C_{i}^{k}}^{*}\right)$. It is clear that $\operatorname{supp}\left(a_{i}^{\prime}\right) \cap \operatorname{supp}\left(b_{i}^{\prime}\right)=\emptyset$.

Suppose that $f_{i}$ is a nonzero polynomial and $\operatorname{supp}\left(a_{i}^{\prime}\right) \neq \emptyset$. If $\operatorname{supp}\left(a_{i}^{\prime}\right)=$ $\{(i-1) k+1, \ldots, i k\}$, then $\operatorname{supp}\left(b_{i}^{\prime}\right)=\emptyset$. From Remark 3.7 we deduce $f_{i} \in J$. If $\operatorname{supp}\left(a_{i}^{\prime}\right) \subsetneq\{(i-1) k+1, \ldots, i k\}$, we know that $\operatorname{supp}\left(b_{i}^{\prime}\right) \neq \emptyset$ and all the coordinates of $a_{i}^{\prime}$ and $b_{i}^{\prime}$ are equal to $\frac{q-1}{2}$. From Proposition 3.9 we get that $\operatorname{supp}\left(a_{i}^{\prime}\right) \cup \operatorname{supp}\left(b_{i}^{\prime}\right)=\{(i-1) k+1, \ldots, i k\}$ and by Proposition 3.4 we find $f_{i} \in J$. In any case we obtain $f_{i} \in J$.

On the other hand we have that:

$$
f^{\prime}=\left(t^{a_{1}^{\prime}}-t^{b_{1}^{\prime}}\right) t^{a_{2}^{\prime}} \cdots t^{a_{m}^{\prime}}+t^{b_{1}^{\prime}}\left[t^{a_{2}^{\prime}} \cdots t^{a_{m}^{\prime}}-t^{b_{2}^{\prime}} \cdots t^{b_{m}^{\prime}}\right] .
$$

If we do the same procedure with the binomial $t^{a_{2}^{\prime}} \cdots t^{a_{m}^{\prime}}-t^{b_{2}^{\prime}} \cdots t^{b_{m}^{\prime}}$, we prove $t^{a_{2}^{\prime}} \cdots t^{a_{m}^{\prime}}-t^{b_{2}^{\prime}} \cdots t^{b_{m}^{\prime}}=\left(t^{a_{2}^{\prime}}-t^{b_{2}^{\prime}}\right) t^{a_{3}^{\prime}} \cdots t^{a_{m}^{\prime}}+t^{b_{2}^{\prime}}\left[t^{a_{3}^{\prime}} \cdots t^{a_{m}^{\prime}}-t^{b_{3}^{\prime}} \cdots t^{b_{m}^{\prime}}\right]$, therefore:

$$
f^{\prime}=f_{1} t_{2}^{a_{2}^{\prime}} \cdots t^{a_{m}^{\prime}}+f_{2} t^{b_{1}^{\prime}} t_{3}^{a_{3}^{\prime}} \cdots t^{a_{m}^{\prime}}+t^{b_{1}^{\prime}} t^{b_{2}^{\prime}}\left[t^{a_{3}^{\prime}} \cdots t_{m}^{a_{m}^{\prime}}-t^{b_{3}^{\prime}} \cdots t^{b_{m}^{\prime}}\right] .
$$

If we proceed like before with the binomial $t^{a_{3}^{\prime}} \cdots t^{a_{m}^{\prime}}-t^{b_{3}^{\prime}} \cdots t^{b_{m}^{\prime}}$ and we continue, we get:

$$
f^{\prime}=\sum_{j=1}^{m} f_{j} h_{j}
$$

where $h_{j} \in K\left[t_{1}, \ldots, t_{k m}\right]$ for all $j$. As $f_{j} \in J$ for all $j$ we show $f^{\prime} \in J$.

Let $\mathcal{G}_{i}$ be a graph with $m_{i}$ connected components and each component is a $k_{i}$-cycle, where $1 \leq i \leq r$. Suppose that $k_{i}=2 \gamma_{i}+1$ and $C_{i 1}^{k_{i}}=x_{1}^{i} \cdots x_{k_{i}}^{i} x_{1}^{i}$, $C_{i 2}^{k_{i}}=x_{k_{i}+1}^{i} \cdots x_{2 k_{i}}^{i} x_{k_{i}+1}^{i}, \ldots, C_{i m_{i}}^{k_{i}}=x_{\left(m_{i}-1\right) k_{i}+1}^{i} \cdots x_{m_{i} k_{i}}^{i} x_{\left(m_{i}-1\right) k_{i}+1}^{i}$ are all the components of $\mathcal{G}_{i}$. Let $\mathcal{G}=\bigcup_{i=1}^{r} \mathcal{G}_{i}$. In this case we will work with the polynomial ring $K\left[t_{1}^{1}, \ldots, t_{k_{1} m_{1}}^{1}, \ldots, t_{1}^{r}, \ldots, t_{k_{r} m_{r}}^{r}\right]$.

For each $1 \leq i \leq r$, let: 


$$
\begin{gathered}
F_{1}^{i}=\left\{A \subseteq\left\{1, \ldots, k_{i}\right\}|| A \mid=\gamma_{i}\right\}, \\
F_{2}^{i}=\left\{A \subseteq\left\{k_{i}+1, \ldots, 2 k_{i}\right\}|| A \mid=\gamma_{i}\right\}, \\
\vdots \\
F_{m_{i}}^{i}=\left\{A \subseteq\left\{\left(m_{i}-1\right) k_{i}+1, \ldots, m_{i} k_{i}\right\}|| A \mid=\gamma_{i}\right\} .
\end{gathered}
$$

Let $A_{j}^{i}=\left\{\left(t_{\alpha_{1}}^{i}\right)^{\frac{q-1}{2}} \cdots\left(t_{\alpha_{\gamma_{i}}}^{i}\right)^{\frac{q-1}{2}}-\left(t_{w_{1}}^{i}\right)^{\frac{q-1}{2}} \cdots\left(t_{w_{\gamma_{i}+1}}^{i}\right)^{\frac{q-1}{2}} \mid\left\{\alpha_{1}, \ldots, \alpha_{\gamma_{i}}\right\} \in\right.$ $F_{j}^{i}$ and $\left.\left\{w_{1}, \ldots, w_{\gamma_{i}+1}\right\}=\left\{(j-1) k_{i}+1, \ldots, j k_{i}\right\}-\left\{\alpha_{1}, \ldots, \alpha_{\gamma_{i}}\right\}\right\}$. Note that $\bigcup_{j=1}^{m_{i}} A_{j}^{i} \subseteq K\left[t_{1}^{i}, \ldots, t_{k_{i} m_{i}}^{i}\right]$. The following theorem is a generalization of Theorem 3.10.

Theorem 3.11. Let $\mathcal{G}=\bigcup_{i=1}^{r} \mathcal{G}_{i}$. Then the vanishing ideal of $X_{\mathcal{G}}^{*}$ is given by:

$$
\mathbf{I}\left(X_{\mathcal{G}}^{*}\right)=\left\langle\bigcup_{i=1}^{r}\left\{\left(t_{j}^{i}\right)^{q-1}-1\right\}_{j=1}^{k_{i} m_{i}} \bigcup\left(\bigcup_{i=1}^{r} \bigcup_{j=1}^{m_{i}} A_{j}^{i}\right)\right\rangle .
$$

Proof. Let $J=\left\langle\bigcup_{i=1}^{r}\left\{\left(t_{j}^{i}\right)^{q-1}-1\right\}_{j=1}^{k_{i} m_{i}} \bigcup\left(\bigcup_{i=1}^{r} \bigcup_{j=1}^{m_{i}} A_{j}^{i}\right)\right\rangle$. The proof is similar to the proof of Theorem 3.10. It follows from Lemma 3.1 that $J \subseteq \mathbf{I}\left(X_{\mathcal{G}}^{*}\right)$. Now we will prove the other inclusion. We know that $\mathbf{I}\left(X_{\mathcal{G}}^{*}\right)$ is generated by binomials, let:

$$
f=t^{a_{1}} \cdots t^{a_{r}}-t^{b_{1}} \cdots t^{b_{r}} \in \mathbf{I}\left(X_{\mathcal{G}}^{*}\right),
$$

where $t^{a_{i}}$ and $t^{b_{i}}$ are monomials in $K\left[t_{1}^{i}, \ldots, t_{k_{i} m_{i}}^{i}\right]$ for all $i$. Note that we can write $f$ as:

$f=$
$\left(t^{a_{i}}-t^{b_{i}}\right) t^{a_{1}} \cdots t^{a_{i-1}} t^{a_{i+1}} \cdots t^{a_{r}}+t^{b_{i}}\left[t^{a_{1}} \cdots t^{a_{i-1}} t^{a_{i+1}}-t^{b_{1}} \cdots t^{b_{i-1}} t^{b_{i+1}} \cdots t^{b_{r}}\right]$.

Let $f_{i}=t^{a_{i}}-t^{b_{i}}$. As $f \in \mathbf{I}\left(X_{\mathcal{G}}^{*}\right)$, it follows that $f_{i} \in \mathbf{I}\left(X_{\mathcal{G}_{i}}^{*}\right)$, from Theorem 3.10 we deduce $f_{i} \in J$. It is easy to see that we can write $f$ as:

$$
f=\sum_{i=1}^{r} f_{i} h_{i}
$$

where $h_{i} \in K\left[t_{1}^{1}, \ldots, t_{k_{1} m_{1}}^{1}, \ldots, t_{1}^{r}, \ldots, t_{k_{r} m_{r}}^{r}\right]$ for all $i$, therefore $f \in J$. 


\section{The Regularity Of The Vanishing Ideal Of Odd Cycles}

We continue with the notation and definitions used in the introduction and in the preliminaries. Let $\mathcal{G}_{i}$ be a graph with $m_{i}$ connected components, suppose that each component is a $k_{i}$-cycle with $k_{i}=2 \gamma_{i}+1$ and $1 \leq i \leq r$. The following proposition is easy to prove.

Proposition 4.1. Let $\mathcal{G}=\bigcup_{i=1}^{r} \mathcal{G}_{i}$. Using the same notation of Theorem 3.11, let:

$$
G=\bigcup_{i=1}^{r}\left\{\left(t_{j}^{i}\right)^{q-1}-1\right\}_{j=1}^{k_{i} m_{i}} \bigcup\left(\bigcup_{i=1}^{r} \bigcup_{j=1}^{m_{i}} A_{j}^{i}\right)
$$

Then $G$ is a Gröbner basis for $\mathbf{I}\left(X_{\mathcal{G}}^{*}\right)$ with respect to grlex order.

Theorem 4.2. Let $\mathcal{G}$ be a graph with $m$ connected components and each component is a $k$-cycle. Suppose that $k=2 \gamma+1$ and let $S=K\left[t_{1}, \ldots, t_{k m}\right]$, then:

$$
\operatorname{reg}\left(S / \mathbf{I}\left(X_{\mathcal{G}}^{*}\right)\right)=m(k+\gamma)\left(\frac{q-1}{2}\right)-k m
$$

Proof. Let $\mathbb{Y}_{\mathcal{G}}^{*}$ be the projective closure of $X_{\mathcal{G}}^{*}$. We know that for each $d \geq$ 1 the codes $C_{X_{\mathcal{G}}^{*}}(d)$ and $C_{\mathbb{Y}_{\mathcal{G}}^{*}}(d)$ have the same basic parameters (see [15, Theorem 2.4]). From Proposition 4.1 we know that:

$$
G=\left\{t_{i}^{q-1}-1\right\}_{i=1}^{k m} \bigcup\left(\bigcup_{i=1}^{m} A_{i}\right)
$$

is a Gröbner basis for $\mathbf{I}\left(X_{\mathcal{G}}^{*}\right)$. On the other hand we know that $\mathbf{I}\left(\mathbb{Y}_{\mathcal{G}}^{*}\right)=\mathbf{I}\left(X_{\mathcal{G}}^{*}\right)^{h}$, where $\mathbf{I}\left(X_{\mathcal{G}}^{*}\right)^{h}$ is the homogenization of $\mathbf{I}\left(X_{\mathcal{G}}^{*}\right)$. We are going to homogenize with respect to the variable $u$, since $G$ is a Gröbner basis for $\mathbf{I}\left(X_{\mathcal{G}}^{*}\right)$ with respect to the grlex order, it follows that $G^{h}$ is a Gröbner basis for $\mathbf{I}\left(X_{\mathcal{G}}^{*}\right)^{h} \subseteq S[u]$, where $S[u]=K\left[t_{1}, \ldots, t_{k m}, u\right]$, regarding the order:

$$
t^{\delta} u^{a}>_{h} t^{\beta} u^{b} \Leftrightarrow t^{\delta}>_{\text {grlex }} t^{\beta} \text { or } t^{\delta}=t^{\beta} \text { and } a>b,
$$

where $t^{\delta}$ and $t^{\beta}$ are monomials in $S$. Denote by $R$ the graded $\operatorname{ring} S[u] /$ $\mathbf{I}\left(X_{\mathcal{G}}^{*}\right)^{h}$. Consider $u \in S[u]$, let $\bar{u}=\mathbf{I}\left(X_{\mathcal{G}}^{*}\right)^{h}+u$. $\bar{u}$ is regular on $R$, then we have the following exact sequence of graded $S[u]$-modules:

$$
0 \rightarrow R[-1] \stackrel{\bar{u}}{\rightarrow} R \rightarrow R /\langle\bar{u}\rangle \rightarrow 0,
$$


where $R[-1]$ is the graded $S[u]$-module obtained by a shift in the graduation, in other words $R[-1]_{i}=R_{i-1}$. Since $S[u] / \mathbf{I}\left(X_{\mathcal{G}}^{*}\right)^{h}$ is a 1-dimensional ring, the regularity of $S[u] / \mathbf{I}\left(X_{\mathcal{G}}^{*}\right)^{h}$ is the least integer $r$ for which $H_{\mathbb{Y}_{\mathcal{G}}^{*}}(d)$ is equal to some constant for all $d \geq r$. From the last exact sequence we have $H_{\mathbb{Y}_{\mathcal{G}}^{*}}(d)-$ $H_{\mathbb{Y}_{\mathcal{G}}^{*}}(d-1)=\operatorname{dim}_{K}(R /\langle\bar{u}\rangle)_{d}$. For $d \geq 1$, we define:

$$
h_{d}:=\operatorname{dim}_{K}(R /\langle\bar{u}\rangle)_{d}=H_{\mathbb{Y}_{\mathcal{G}}^{*}}(d)-H_{\mathbb{Y}_{\mathcal{G}}^{*}}(d-1) .
$$

First we will prove that $\operatorname{reg}\left(S[u] / \mathbf{I}\left(X_{\mathcal{G}}^{*}\right)^{h}\right) \leq m(k+\gamma)\left(\frac{q-1}{2}\right)-k m$. Let $\alpha=m(k+\gamma)\left(\frac{q-1}{2}\right)-k m$. If we show that $h_{d}=0$ for $d \geq \alpha+1$, then $H_{\mathbb{Y}_{\mathcal{G}}^{*}}(d-1)=H_{\mathbb{Y}_{\mathcal{G}}^{*}}(d)$ for $d-1 \geq \alpha$, and our result follows. Let $d \geq \alpha+1$. To show that $h_{d}=0$ for $d \geq \alpha+1$, it is enough to prove that if $g \in S[u]_{d}$ is a monomial, then:

$$
\langle\bar{u}\rangle+\left(\mathbf{I}\left(X_{\mathcal{G}}^{*}\right)^{h}+g\right)=\langle\bar{u}\rangle+\left(\mathbf{I}\left(X_{\mathcal{G}}^{*}\right)^{h}\right) .
$$

Let $g=t^{a_{1}} \cdots t^{a_{m}} u^{a_{0}} \in S[u]_{d}$, where $t^{a_{i}}$ is a monomial in $K\left[t_{(i-1) k+1}, \ldots\right.$, $\left.t_{i k}\right]$ for $i=1, \ldots, m$. If $a_{0}>0$, it is clear that 3 follows, therefore we will suppose that $a_{0}=0$. For $i \in\{1, \ldots, m\}$, let $g_{i}=t^{a_{1}} \cdots t^{a_{i-1}} t^{a_{i+1}} \cdots t^{a_{m}}$.

Let $i \in\{1, \ldots, m\}$, suppose that there is $w \in\{(i-1) k+1, \ldots, i k\}$ such that $t_{w}^{q-1} \mid t^{a_{i}}$, then $t^{a_{i}}=t_{w}^{q-1} t^{c}$, where $t^{c}$ is a monomial in $K\left[t_{(i-1) k+1}, \ldots, t_{i k}\right]$. Therefore we can write $g$ as:

$$
g=t^{a_{1}} \cdots t^{a_{i-1}} t_{w}^{q-1} t^{c} t^{a_{i+1}} \cdots t^{a_{m}}=g_{i} t^{c}\left[t_{w}^{q-1}-u^{q-1}\right]+u^{q-1} g_{i} t^{c},
$$

it is clear that 3 follows, then we will suppose that all the coordinates of each $a_{i}$ are less or equal than $q-2$. Now we will suppose that for each $i \in\{1, \ldots, m\}$, the monomial $t^{a_{i}}$ is not divisible by any $L T(f)$, for all $f \in A_{i}^{h}$. Then we can write $t^{a_{i}}$ as:

$$
t^{a_{i}}=\left(t_{w_{i 1}}^{a_{i 1}} \cdots t_{w_{i \gamma+1}}^{a_{i \gamma+1}}\right) t_{w_{i \gamma+2}}^{a_{i \gamma+2}} \cdots t_{w_{i k}}^{a_{i k}},
$$

where $0 \leq a_{i j} \leq \frac{q-1}{2}-1$ for all $(i, j) \in\{1, \ldots, m\} \times\{1, \ldots, \gamma+1\}$ and $\left|\left\{w_{i 1}, \ldots, w_{i k}\right\}\right|=k$. On the other hand we have that:

$$
\operatorname{deg}(g)=\sum_{i=1}^{m} \sum_{j=1}^{\gamma+1} a_{i j}+\sum_{i=1}^{m} \sum_{j=\gamma+2}^{k} a_{i j}
$$

it follows that $\operatorname{deg}(g) \leq m(\gamma+1)\left(\frac{q-1}{2}-1\right)+m \gamma(q-2)$, therefore: 


$$
\alpha+1=m(k+\gamma)\left(\frac{q-1}{2}\right)-k m+1 \leq m(\gamma+1)\left(\frac{q-1}{2}-1\right)+m \gamma(q-2),
$$

we deduce that:

$$
\begin{aligned}
m k\left(\frac{q-1}{2}\right)-k m+1 & \leq m\left(\frac{q-1}{2}\right)-m(\gamma+1)+m \gamma(q-2), \\
m\left(\frac{q-1}{2}\right)(k-1)+1 & \leq m[k-(\gamma+1)]+m \gamma(q-2), \\
m \gamma(q-1)+1 & \leq m \gamma+m \gamma(q-2), \\
m \gamma(q-1)+1 & \leq m \gamma(q-1), \\
1 & \leq 0,
\end{aligned}
$$

this is a contradiction, therefore there is $i \in\{1, \ldots, m\}$ such that $t^{a_{i}}$ is divisible by $L T(f)$ for some $f=t_{\alpha_{1}^{2}}^{\frac{q-1}{2}} \cdots t_{\alpha_{\gamma}}^{\frac{q-1}{2}} u^{\frac{q-1}{2}}-t_{w_{1}^{2}}^{\frac{q-1}{2}} \cdots t_{w_{\gamma+1}}^{\frac{q-1}{2}} \in A_{i}^{h}$. Then $t^{a_{i}}=$ $\left(t_{w_{1}^{2}}^{\frac{q-1}{2}} \cdots t_{w_{\gamma+1}^{2}}^{\frac{q-1}{2}}\right) t^{c}$, where $t^{c}$ is a monomial in $K\left[t_{(i-1) k+1}, \ldots, t_{i k}\right]$ and all the coordinates of $c$ are between 0 and $q-2$. We can write $g$ as:

$$
g=-g_{i} t^{c} f+g_{i} t^{c}\left(t_{\alpha_{1}^{2}}^{\frac{q-1}{2}} \cdots t_{\alpha_{\gamma}}^{\frac{q-1}{2}} u^{\frac{q-1}{2}}\right),
$$

then we show 3 follows, thus we have proved that $\operatorname{reg}\left(S[u] / \mathbf{I}\left(X_{\mathcal{G}}^{*}\right)^{h}\right) \leq \alpha$.

Now we will show that $\alpha \leq \operatorname{reg}\left(S[u] / \mathbf{I}\left(X_{\mathcal{G}}^{*}\right)^{h}\right)$. If we show that $h_{d}>0$ for $d=\alpha$, then $H_{\mathbb{Y}_{\mathcal{G}}^{*}}(d-1)<H_{\mathbb{Y}_{\mathcal{G}}^{*}}(d)$, for $d=\alpha$, and our result follows. It suffices to find a monomial $M \in S[u]_{d}$ such that:

$$
\langle\bar{u}\rangle+\left(\mathbf{I}\left(X_{\mathcal{G}}^{*}\right)^{h}+M\right) \neq\langle\bar{u}\rangle+\left(\mathbf{I}\left(X_{\mathcal{G}}^{*}\right)^{h}\right) .
$$

For $i \in\{1, \ldots, m\}$, let $M_{i}=t_{(i-1) k+1}^{q-2} \cdots t_{(i-1) k+\gamma}^{q-2} t_{(i-1) k+\gamma+1}^{\frac{q-1}{2}-1} \cdots t_{i k}^{\frac{q-1}{2}-1}$. Note that $\operatorname{deg}\left(M_{i}\right)=(k+\gamma)\left(\frac{q-1}{2}\right)-k$ and $M_{i}$ is not divisible by any $L T(f)$ for all $f \in G_{i}^{h}$, where $G_{i}=\left\{t_{j}^{q-1}-1\right\}_{j=(i-1) k+1}^{i k} \cup A_{i}$. Let:

$$
M=\prod_{i=1}^{m} M_{i}
$$

It is clear that $M \in S[u]_{d}$. Suppose that:

$$
\langle\bar{u}\rangle+\left(\mathbf{I}\left(X_{\mathcal{G}}^{*}\right)^{h}+M\right)=\langle\bar{u}\rangle+\left(\mathbf{I}\left(X_{\mathcal{G}}^{*}\right)^{h}\right),
$$

then we get that $\mathbf{I}\left(X_{\mathcal{G}}^{*}\right)^{h}+M \in\langle\bar{u}\rangle$, therefore $\mathbf{I}\left(X_{\mathcal{G}}^{*}\right)^{h}+M=\mathbf{I}\left(X_{\mathcal{G}}^{*}\right)^{h}+\tilde{g} u$, where $\tilde{g} \in S[u]$, thus we deduce that $M-\tilde{g} u \in \mathbf{I}\left(X_{\mathcal{G}}^{*}\right)^{h}$, then we can write $M$ as: 


$$
M=g^{\prime}+\tilde{g} u,
$$

where $g^{\prime} \in \mathbf{I}\left(X_{\mathcal{G}}^{*}\right)^{h}$, making $u=0$ in the previous equation we obtain:

$$
M \in\left\langle\left\{L T(f) \mid f \in G^{h}\right\}\right\rangle,
$$

it follows that $M$ is divisible by $L T(f)$ for some $f \in G^{h}$, and this is a contradiction, thus 4 follows.

Theorem 4.3. Let $\mathcal{G}_{i}$ be a graph with $m_{i}$ connected components, suppose that each component is a $k_{i}$-cycle with $k_{i}=2 \gamma_{i}+1$ and $1 \leq i \leq l$. We will work with the polynomial ring $\tilde{S}=K\left[t_{1}^{1}, \ldots, t_{k_{1} m_{1}}^{1}, \ldots, t_{1}^{l}, \ldots, t_{k_{l} m_{l}}^{l}\right]$. Let $\mathcal{G}=\bigcup_{i=1}^{l} \mathcal{G}_{i}$, then:

$$
\operatorname{reg}\left(\tilde{S} / \mathbf{I}\left(X_{\mathcal{G}}^{*}\right)\right)=\sum_{i=1}^{l} m_{i}\left(k_{i}+\gamma_{i}\right)\left(\frac{q-1}{2}\right)-k_{i} m_{i}
$$

Proof. We will proceed by induction on $l$. If $l=1$ there is nothing else to do. We are going to suppose that our result follows for $l=r$ and we will prove the formula for $l=r+1$. Let $\mathcal{G}^{\prime}=\bigcup_{i=1}^{r} \mathcal{G}_{i}$ and $\mathbb{Y}^{\prime}$ be the projective closure of $X_{\mathcal{G}^{\prime}}^{*}$. Let $S^{\prime}=K\left[t_{1}^{1}, \ldots, t_{k_{1} m_{1}}^{1}, \ldots, t_{1}^{r}, \ldots, t_{k_{r} m_{r}}^{r}\right]$, in the projective space we will work with the ring $S^{\prime}[u]$. We know that the the Hilbert series of $S^{\prime}[u] / \mathbf{I}\left(\mathbb{Y}^{\prime}\right)$ is given by:

$$
F_{\mathbb{Y}^{\prime}}(t)=\frac{f(t)}{1-t},
$$

where $\operatorname{deg}(f)=\operatorname{reg}\left(S^{\prime}[u] / \mathbf{I}\left(\mathbb{Y}^{\prime}\right)\right)$ and $F\left(S^{\prime}[u] /\left(u, \mathbf{I}\left(\mathbb{Y}^{\prime}\right)\right), t\right)=f(t)$. Let $\mathbb{Y}^{\prime \prime}$ be the projective closure of $X_{\mathcal{S}_{r+1}}^{*}$ and $S^{\prime \prime}=K\left[t_{1}^{r+1}, \ldots, t_{k_{r+1} m_{r+1}}^{r+1}\right]$. We know that the Hilbert series of $S^{\prime \prime}[u] / \mathbf{I}\left(\mathbb{Y}^{\prime \prime}\right)$ is given by:

$$
F_{\mathbb{Y}^{\prime \prime}}(t)=\frac{g(t)}{1-t},
$$

where $\operatorname{deg}(g)=\operatorname{reg}\left(S^{\prime \prime}[u] / \mathbf{I}\left(\mathbb{Y}^{\prime \prime}\right)\right)$ and $F\left(S^{\prime \prime}[u] /\left(u, \mathbf{I}\left(\mathbb{Y}^{\prime \prime}\right)\right), t\right)=g(t)$. According to [22, Proposition 2.2.20, p.42], we have an isomorphism:

$$
S^{\prime}[u] /\left(u, \mathbf{I}\left(\mathbb{Y}^{\prime}\right)\right) \otimes_{K} S^{\prime \prime}[u] /\left(u, \mathbf{I}\left(\mathbb{Y}^{\prime \prime}\right)\right) \cong S[u] /(u, \mathbf{I}(\mathbb{Y})),
$$

where $S=K\left[t_{1}^{1}, \ldots, t_{k_{1} m_{1}}^{1}, \ldots, t_{1}^{r+1}, \ldots, t_{k_{r+1} m_{r+1}}^{r+1}\right]$ and $\mathbb{Y}$ is the projective closure of $X_{\mathcal{G}}^{*}$ with $\mathcal{G}=\bigcup_{i=1}^{r+1} \mathcal{G}_{i}$. On the other hand we have that $F\left(S^{\prime}[u] /\right.$ $\left.\left(u, \mathbf{I}\left(\mathbb{Y}^{\prime}\right)\right) \otimes_{K} S^{\prime \prime}[u] /\left(u, \mathbf{I}\left(\mathbb{Y}^{\prime \prime}\right)\right), t\right)=F(S[u] /(u, \mathbf{I}(\mathbb{Y})), t)$, thus:

$$
F(S[u] /(u, \mathbf{I}(\mathbb{Y})), t)=F\left(S^{\prime}[u] /\left(u, \mathbf{I}\left(\mathbb{Y}^{\prime}\right)\right), t\right) F\left(S^{\prime \prime}[u] /\left(u, \mathbf{I}\left(\mathbb{Y}^{\prime \prime}\right)\right), t\right),
$$


(see [22, p.102] ) therefore $F(S[u] /(u, \mathbf{I}(\mathbb{Y})), t)=f(f) g(t)$, it follows that $\operatorname{reg}(S[u] / \mathbf{I}(\mathbb{Y}))=\operatorname{reg}\left(S^{\prime}[u] / \mathbf{I}\left(\mathbb{Y}^{\prime}\right)\right)+\operatorname{reg}\left(S^{\prime \prime}[u] / \mathbf{I}\left(\mathbb{Y}^{\prime \prime}\right)\right)$. If we apply inductive hypothesis, our result follows.

\section{Dimension Of Parameterized affine Codes by Odd Cy- cles}

Let $\mathcal{G}$ be a $k$-cycle and $S=K\left[t_{1}, \ldots, t_{k}\right]$, suppose that $k=2 \gamma+1$. Let $F=\{A \subseteq\{1, \ldots, k\}|| A \mid=\gamma\}$ and $d \geq 1$. For $H=\left\{h_{1}, \ldots, h_{\gamma}\right\} \in F$, let:

$$
\begin{gathered}
A_{H}(d)=\left\{t_{h_{1}}^{a_{h_{1}}} \cdots t_{h_{\gamma}}^{a_{h_{\gamma}}} t_{w_{1}}^{a_{w_{1}}} \cdots t_{w_{\gamma+1}}^{a_{w_{\gamma+1}}} \mid\left\{w_{1}, \ldots, w_{\gamma+1}\right\}=\{1, \ldots, k\}-H,\right. \\
\left.a_{h_{i}}<q-1 \text { for all } i, a_{w_{j}}<\frac{q-1}{2} \text { for all } j \text { and } \sum_{i=1}^{\gamma} a_{h_{i}}+\sum_{j=1}^{\gamma+1} a_{w_{j}} \leq d\right\} .
\end{gathered}
$$

Let $r=|F|=\left(\begin{array}{l}k \\ \gamma\end{array}\right)$ and $F=\left\{H_{1}, \ldots, H_{r}\right\}$. The Hilbert function of $\mathbf{I}\left(X_{\mathcal{G}}^{*}\right)$ can be obtained from the following result.

Theorem 5.1. Let $\mathcal{G}$ be a $k$-cycle and $S=K\left[t_{1}, \ldots, t_{k}\right]$, suppose that $k=$ $2 \gamma+1$. Using the above notation, we have that:

$$
H_{X_{\mathcal{G}}^{*}}(d)=\left|\bigcup_{i=1}^{r} A_{H_{i}}(d)\right| .
$$

Proof. Let $A=\left\{t_{w_{1}^{2}}^{\frac{q-1}{2}} \cdots t_{w_{\gamma+1}}^{\frac{q-1}{2}}-t_{\alpha_{1}^{\frac{q-1}{2}}}^{\frac{q-1}{\frac{q-1}{2}}} \mid\left\{\alpha_{1}, \ldots, \alpha_{\gamma}\right\} \in F\right.$ and $\left\{w_{1}, \ldots\right.$, $\left.\left.w_{\gamma+1}\right\}=\{1, \ldots, k\} \backslash\left\{\alpha_{1}, \ldots, \alpha_{\gamma}\right\}\right\}$. We know that the vanishing ideal of $X_{\mathcal{G}}^{*}$ is given by:

$$
\mathbf{I}\left(X_{\mathcal{G}}^{*}\right)=\left\langle\left\{t_{i}^{q-1}-1\right\}_{i=1}^{k} \cup A\right\rangle .
$$

Let $G=\left\{t_{i}^{q-1}-1\right\}_{i=1}^{k} \cup A$, by Proposition $4.1, G$ is a Gröbner basis for $\mathbf{I}\left(X_{\mathcal{G}}^{*}\right)$ with respect to grlex order. Let $d \geq 1$, we know that $H_{X_{\mathcal{G}}^{*}}(d)$ is the number of standard monomials of degree less or equal to $d$. Let:

$$
\Delta_{\mathbf{I}\left(X_{\mathcal{G}}^{*}\right)}(d)=\left\{m \in \Delta_{>_{\text {grlex }}}\left(\mathbf{I}\left(X_{\mathcal{G}}^{*}\right)\right) \mid \operatorname{deg}(m) \leq d\right\}
$$

we are going to prove that:

$$
\Delta_{\mathbf{I}\left(X_{\mathcal{G}}^{*}\right)}(d)=\bigcup_{i=1}^{r} A_{H_{i}}(d) .
$$


Let $m \in \Delta_{\mathbf{I}\left(X_{\mathcal{G}}^{*}\right)}(d)$, then $m \in \Delta_{>_{\text {grlex }}}\left(\mathbf{I}\left(X_{\mathcal{G}}^{*}\right)\right)$ and $\operatorname{deg}(m) \leq d$. As $m \in$ $\Delta_{>_{\text {grlex }}}\left(\mathbf{I}\left(X_{\mathcal{G}}^{*}\right)\right)$, it follows that $m \notin\left\langle L T\left(\mathbf{I}\left(X_{\mathcal{G}}^{*}\right)\right)\right\rangle$. On the other hand $G$ is a Gröbner basis, then $m$ is not divisible by any leader monomial of any element of $G$. Therefore, there is $H=\left\{\alpha_{1}, \ldots, \alpha_{\gamma}\right\} \in F$ such that:

$$
m=t_{\alpha_{1}}^{a_{\alpha_{1}}} \cdots t_{\alpha_{\gamma}}^{a_{\alpha_{\gamma}}} t_{w_{1}}^{a_{w_{1}}} \cdots t_{w_{\gamma+1}}^{a_{w_{\gamma+1}}},
$$

where $\left\{w_{1}, \ldots, w_{\gamma+1}\right\}=\{1, \ldots, k\} \backslash H, a_{\alpha_{i}}<q-1$ for all $i$ and $a_{w_{j}}<\frac{q-1}{2}$ for all $j$. It follows that $m \in A_{H}(d)$, thus:

$$
\Delta_{\mathbf{I}\left(X_{\Im}^{*}\right)}(d) \subseteq \bigcup_{i=1}^{r} A_{H_{i}}(d)
$$

The other inclusion is clear.

For $d \geq 1$ we define the following sets:

$A_{\gamma}(d)=\left\{t_{1}^{a_{1}} \cdots t_{\gamma}^{a_{\gamma}} t_{\gamma+1}^{a_{\gamma+1}} \cdots t_{k}^{a_{k}} \mid 0 \leq a_{i}<q-1\right.$ for all $i=1, \ldots, \gamma$, $0 \leq a_{j}<\frac{q-1}{2}$ for all $j=\gamma+1, \ldots, k$ and $\left.\sum_{i=1}^{k} a_{i} \leq d\right\}$,

$A_{\gamma-1}(d)=\left\{t_{1}^{a_{1}} \cdots t_{\gamma-1}^{a_{\gamma-1}} t_{\gamma}^{a_{\gamma}} \cdots t_{k}^{a_{k}} \mid 0 \leq a_{i}<q-1\right.$ for all $i=1, \ldots, \gamma-1$, $0 \leq a_{j}<\frac{q-1}{2}$ for all $j=\gamma, \ldots, k$ and $\left.\sum_{i=1}^{k} a_{i} \leq d\right\}$,

$A_{1}(d)=\left\{t_{1}^{a_{1}} t_{2}^{a_{2}} \cdots t_{k}^{a_{k}} \mid 0 \leq a_{1}<q-1,0 \leq a_{j}<\frac{q-1}{2}\right.$ for all $j=2, \ldots, k$ and $\left.\sum_{i=1}^{k} a_{i} \leq d\right\}$,

$$
A_{0}(d)=\left\{t_{1}^{a_{1}} \cdots t_{k}^{a_{k}} \mid 0 \leq a_{i}<\frac{q-1}{2} \text { for all } i=1, \ldots, k \text { and } \sum_{i=1}^{k} a_{i} \leq d\right\} .
$$

Remark 5.2. Let $1 \leq l \leq r$ and $1 \leq i_{1}<\cdots<i_{l} \leq r$. It is easy to see that:

$$
\left|A_{H_{i_{1}}}(d) \cap \cdots \cap A_{H_{i_{l}}}(d)\right| \in\left\{\left|A_{\gamma}(d)\right|, \ldots,\left|A_{1}(d)\right|,\left|A_{0}(d)\right|\right\} .
$$

Therefore if we use Theorem 5.1, we can write $H_{X_{9}^{*}}(d)$ as:

$$
H_{X_{\mathcal{G}}^{*}}(d)=\beta_{0}\left|A_{0}(d)\right|+\beta_{1}\left|A_{1}(d)\right|+\cdots+\beta_{\gamma}\left|A_{\gamma}(d)\right|,
$$

where $\beta_{0}, \ldots, \beta_{\gamma}$ are integers independent of $d$ and $q$.

Proposition 5.3. Let $0<i \leq \gamma$ and $S=K\left[t_{1}, \ldots, t_{k}\right]$. Let $X_{i}^{*}=\left\{\left(x_{1}, \ldots, x_{i}\right.\right.$, $\left.x_{i+1}^{2}, \ldots, x_{k}^{2}\right) \mid x_{j} \in K^{*}$ for all $\left.j\right\}$ and $X_{0}^{*}=\left\{\left(x_{1}^{2}, \ldots, x_{k}^{2}\right) \mid x_{i} \in K^{*}\right.$ for all $\left.i\right\}$. Then: 
(i) $H_{X_{i}^{*}}(d)=\left|A_{i}(d)\right|$.

(ii) $H_{X_{0}^{*}}(d)=\left|A_{0}(d)\right|$.

Proof. We are going to prove (i). First, we are going to prove that:

$$
\mathbf{I}\left(X_{i}^{*}\right)=\left\langle t_{1}^{q-1}-1, \ldots, t_{i}^{q-1}-1, t_{i+1}^{\frac{q-1}{2}}-1, \ldots, t_{k}^{\frac{q-1}{2}}-1\right\rangle .
$$

Let $>_{\text {grlex }}$ be the grlex order on $S$ and let $f \in \mathbf{I}\left(X_{i}^{*}\right)$. By the division algorithm (see [3, Theorem 3,p. 64]) we can write $f$ as:

$$
f=\sum_{j=1}^{i} h_{j}\left(t_{j}^{q-1}-1\right)+\sum_{j=i+1}^{k} h_{j}\left(t_{j}^{\frac{q-1}{2}}-1\right)+G\left(t_{1}, \ldots, t_{k}\right),
$$

where $h_{j} \in S$ for all $j$ and none term of $G$ is divisible by any $t_{1}^{q-1}, \ldots, t_{i}^{q-1}, t_{i+1}^{\frac{q-1}{2}}$, $\ldots, t_{k}^{\frac{q-1}{2}}$. By Combinatorial-Nullstellensatz, taking $S_{j}=K^{*}$ for all $j=$ $1, \ldots, i$ and $S_{j}=\left\{a^{2} \mid a \in K^{*}\right\}$ for all $j=i+1, \ldots, k$, we obtain $G=0$. Therefore:

$$
\mathbf{I}\left(X_{i}^{*}\right) \subseteq\left\langle t_{1}^{q-1}-1, \ldots, t_{i}^{q-1}-1, t_{i+1}^{\frac{q-1}{2}}-1, \ldots, t_{k}^{\frac{q-1}{2}}-1\right\rangle .
$$

The other inclusion is clear. Let $G=\left\{t_{1}^{q-1}-1, \ldots, t_{i}^{q-1}-1, t_{i+1}^{\frac{q-1}{2}}-\right.$ $\left.1, \ldots, t_{k}^{\frac{q-1}{2}}-1\right\}$, by [3, Theorem 6,p. 85], it follows that $G$ is a Gröbner basis for $\mathbf{I}\left(X_{i}^{*}\right)$ with respect to grlex order. For $d \geq 1$ we know that $H_{X_{i}^{*}}(d)$ is the number of standard monomials of degree less or equal to $d$, thus (i) follows. The proof of (ii) is similar.

The sets $X_{0}^{*}, \ldots, X_{\gamma}^{*}$ in Proposition 5.3 are degenerate torus (see [14, Section 4]). From Remark 5.2 and Proposition 5.3 we get that the Hilbert function of $\mathbf{I}\left(X_{\mathcal{G}}^{*}\right)$ can be written as:

$$
H_{X_{S}^{*}}(d)=\beta_{0} H_{X_{0}^{*}}(d)+\cdots+\beta_{\gamma} H_{X_{\gamma}^{*}}(d),
$$

where $\beta_{0}, \ldots, \beta_{\gamma}$ are integers independent of $d$ and $q$. In other words, we can write $H_{X_{\mathcal{S}}^{*}}(d)$ as linear combination of Hilbert functions of degenerate torus. For each $i \in\{0, \ldots, \gamma\}$ we can find an explicit formula for $H_{X_{i}^{*}}(d)$ in [14, Section 4]; therefore if we want to find an explicit formula for $H_{X_{g}^{*}}(d)$, we just need to find the values of $\beta_{0}, \ldots, \beta_{\gamma}$.

Example 5.4. Let $K=\mathbb{F}_{5}$ and $S=K\left[t_{1}, t_{2}, t_{3}, t_{4}, t_{5}\right]$. Let:

$$
X_{\mathcal{G}}^{*}=\left\{\left(x_{1} x_{2}, x_{2} x_{3}, x_{3} x_{4}, x_{4} x_{5}, x_{5} x_{1}\right) \mid x_{i} \in \mathbb{F}_{5}^{*}\right\} .
$$


Let $X_{0}^{*}, X_{1}^{*}, X_{2}^{*}$ be the sets defined in Proposition 5.3. Using Macaulay 2.0, we obtain:

\begin{tabular}{c|c|c|c|c}
$d$ & $H_{X_{2}^{*}}(d)$ & $H_{X_{1}^{*}}(d)$ & $H_{X_{0}^{*}}(d)$ & $H_{X_{9}^{*}}(d)$ \\
\hline 1 & 6 & 6 & 6 & 6 \\
\hline 2 & 18 & 17 & 16 & 21 \\
\hline 9 & 128 & 64 & 32 & 512
\end{tabular}

Note that $\operatorname{reg}\left(S / \mathbf{I}\left(X_{\mathcal{G}}^{*}\right)\right)=9$. Then we have the following system of equations:

$$
\begin{gathered}
6=6 \beta_{2}+6 \beta_{1}+6 \beta_{0} \\
21=18 \beta_{2}+17 \beta_{1}+16 \beta_{0} \\
512=128 \beta_{2}+64 \beta_{1}+32 \beta_{0}
\end{gathered}
$$

Resolving the previous system, we obtain $\beta_{2}=10, \beta_{1}=-15$ and $\beta_{0}=6$. Using Macaulay 2.0 we can verify that for $d \geq 1$ it follows the following equality:

$$
H_{X_{9}^{*}}(d)=10 H_{X_{2}^{*}}(d)-15 H_{X_{1}^{*}}(d)+6 H_{X_{0}^{*}}(d) .
$$

If we change the field $K$, then the last equality will remain true.

\section{References}

[1] N. Alon, Combinatorial Nullstellensatz, Recent trends in combinatorics (Matraháza, 1995), Combin. Probab. Comput. 8 (1999), no. 1-2, 7-29.

[2] W. W. Adams and P. Loustaunau, An Introduction to Gröbner Bases, GSM 3, American Mathematical Society, 1994.

[3] D. Cox, J. Little and D. O'Shea, Ideals, Varieties, and Algorithms, Springer-Verlag, 1992.

[4] P. Delsarte, J. M. Goethals and F. J. MacWilliams, On generalized ReedMuller codes and their relatives, Information and Control 16 (1970), 403442.

[5] I. M. Duursma, C. Rentería and H. Tapia-Recillas, Reed-Muller codes on complete intersections, Appl. Algebra Engrg. Comm. Comput. 11 (2001), no. $6,455-462$. 
[6] D. Eisenbud, The geometry of syzygies: A second course in commutative algebra and algebraic geometry, Graduate texts in Mathematics 229, Springer-Verlag, New York, 2005.

[7] D. Eisenbud and B. Sturmfels, Binomial ideals, Duke Math. J. 84 (1996), $1-45$.

[8] A. V. Geramita, M. Kreuzer and L. Robbiano, Cayley-Bacharach schemesand their canonical modules, Trans. Amer. Math. Soc. 339 (1993), no. $1,163-189$.

[9] L. Gold, J. Little and H. Schenck, Cayley-Bacharach and evaluation codes on complete intersections, J. Pure Appl. Algebra 196 (2005), no. 1, 91-99.

[10] M. González-Sarabia, C. Rentería and H. Tapia-Recillas, Reed-Mullertype codes over the Segre variety, Finite Fields Appl. 8 (2002), no. 4, $511-518$.

[11] M. González-Sarabia and C. Rentería, Evaluation codes associated to complete bipartite graphs, Int. J. Algebra 2 (2008), no. 1-4, 163-170.

[12] M. González-Sarabia, J. Nava, C. Rentería and E. Sarmiento, Parameterized codes over cycles, An. St. Univ. Ovidius Constanta 21 (2013), no. 3, $241-255$.

[13] J. Harris, Algebraic Geometry. A first course, Graduate Texts in Mathematics 133, Springer-Verlag, New York, 1992.

[14] H. H. López, C. Rentería and R. H. Villarreal, Affine cartesian codes, Des. Codes Cryptography. 71 (2014), no. 1, 5-19.

[15] H. H. López, E. Sarmiento, M. Vaz Pinto and R. H. Villarreal, Parameterized affine codes, Studia Sci. Math. Hungar. 49 (2012), no. 3, 406-418.

[16] J. Neves, M. Vaz Pinto and R. H. Villarreal, Vanishing ideals over graphs and even cycles, Comm. Algebra 43 (2015), no. 3, 1050-1075.

[17] C. Rentería, A. Simis and R. H. Villarreal, Algebraic methods for parameterized codes and invariants of vanishing ideals over finite fields, Finite Fields Appl. 17 (2011), no. 1, 81-104.

[18] A. Sørensen, Projective Reed-Muller codes, IEEE Trans. Inform. Theory 37 (1991), no. 6, 1567-1576.

[19] R. Stanley, Hilbert functions of graded algebras, Adv. Math. 28 (1978), 57-83. 
[20] H. Stichtenoth, Algebraic function fields and codes. Universitext, Springer-Verlag, Berlin, 1993.

[21] M. Tsfasman, S. Vladut and D. Nogin, Algebraic geometric codes: basic notions, Mathematical Surveys and Monographs 139, American Mathematical Society, Providence, RI, 2007.

[22] R. H. Villarreal, Monomial Algebras, Monographs and Textbooks in Pure and Applied Mathematics 238, Marcel Dekker, New York, 2001.

M. Eduardo Uribe-Paczka,

Department of Mathematics,

Escuela Superior de Física y Matemáticas,

Instituto Politécnico Nacional, 07300 Mexico City.

Email: muribep1700@alumno.ipn.mx

Eliseo Sarmiento,

Department of Mathematics,

Escuela Superior de Física y Matemáticas,

Instituto Politécnico Nacional, 07300 Mexico City.

Email:eliseo@esfm.ipn.mx

Carlos Rentería Márquez,

Department of Mathematics,

Escuela Superior de Física y Matemáticas,

Instituto Politécnico Nacional, 07300 Mexico City.

Email:renteri@esfm.ipn.mx 